\title{
A Framework for Mapping with Biobotic Insect Networks: From Local to Global Maps ${ }^{\text {tr }}$
}

\author{
Alireza Dirafzoon ${ }^{\mathrm{a}, *}$, Alper Bozkurt ${ }^{\mathrm{a}}$, Edgar Lobaton ${ }^{\mathrm{a}, *}$ \\ a Department of Electrical and Computer Engineering, \\ North Carolina State University, Raleigh, NC 27606, USA
}

\begin{abstract}
We present an approach for global exploration and mapping of unknown environments using a swarm of cyborg insects, known as biobots, for emergency response scenarios under minimal sensing and localization constraints. We exploit natural stochastic motion models and controlled locomotion of biobots in conjunction with an aerial leader to explore and map a domain of interest. A sliding window strategy is adopted to construct local maps from coordinate free encounter information of the agents by means of local metric estimation. Robust topological features from these local representations are extracted using topological data analysis and a classification scheme. These maps are then merged into a global map which can be visualized using a graphical representation, that integrates geometric as well as topological features of the environment. Simulation and experimental results with biologically inspired robotic platform are presented to illustrate and verify the correctness of our approach, which provides building blocks for SLAM with biobotic insects.
\end{abstract}

Keywords: Topological Mapping, Metric Estimation, Manifold Learning, Cyborg Insects, Topological Data Analysis, Emergency Response

\section{Introduction}

Mapping of unknown environments is an essential and challenging task in search and rescue for emergency response application. In particular, teams of small autonomous agents (e.g. biologically inspired milli-robots [1] or cyborg insects known as biobots [2]) have certain advantages over traditional platforms when performing exploration and mapping tasks since larger robotic systems may not be able to reach safely to locations under the rubble of collapsed buildings. Furthermore, biobotic platforms can be particularly versatile at locomotion and navigation in unstructured and dynamic scenes due to their natural ability to crawl through small spaces. Figure 1 shows a realization of a system composed

\footnotetext{
This work was supported by the National Science Foundation under award CNS-1239243.

* Corresponding authors

Email addresses: adirafz@ncsu.edu (Alireza Dirafzoon), aybozkur@ncsu.edu (Alper Bozkurt), ejlobato@ncsu.edu (Edgar Lobaton)
}

of traditional robotic and biobotic agents. Recent developments in neural engineering and neuromuscular stimulation have enabled us to directly control insect locomotion using wireless neurostimulators to enable remotely controlled biobots [2]. Each agent is equipped with system-on-chip based ZigBee enabled wireless neuro-stimulation backpack system and remote navigational control circuits. In particular, we have been able to control Gromphadorhina portentosa (Madagascar hissing) cockroaches in open and mazed environments, and have demonstrated the implementation of fenceless virtual boundaries for such agents $[2,3]$. Mapping and localization in under-rubble environments using such platforms becomes extremely challenging due to hardware limitations and the unstructured nature of the environment. Power and computational resource constraints prohibit us from using traditional on-board imaging techniques for their localization (e.g., visual SLAM $[4,5]$ ). Furthermore, traditional signal propagation based localization (e.g., GPS, or computing signal strength or time of flight $[6,7]$ ) may be unreliable for indoor or 


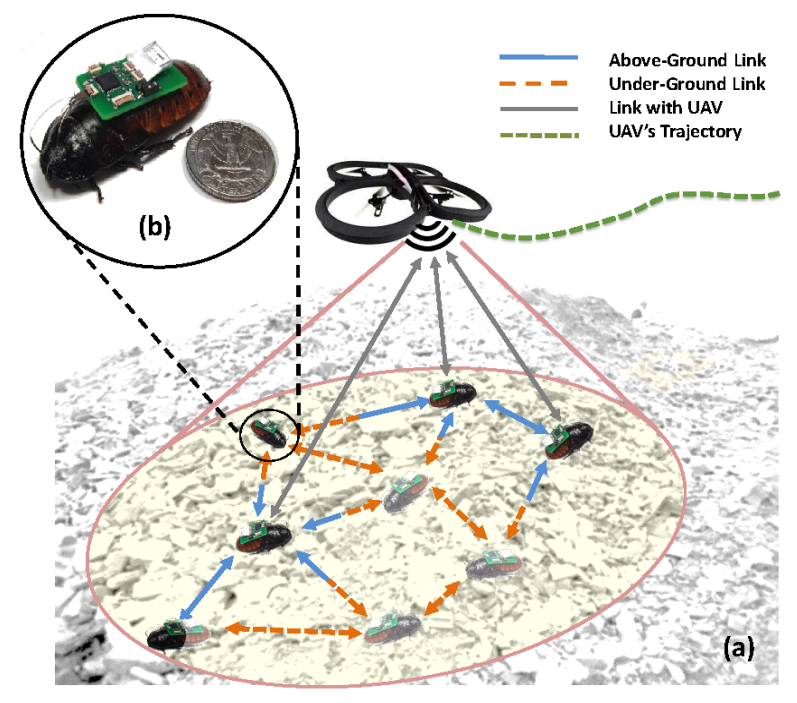

Figure 1: (a) Schematic of a physical environment with a swarm of above-ground and underground biobotic insects in conjunction with an aerial leader for exploration and mapping; underground agents are shown in pale colors, and local communication links are plotted with color-coded arrows. (b) Gromphadorhina portentosa "biobot" with stimulation backpack and battery [2].

underground locations in cluttered environments, and odometry and inertial-based approaches (e.g., [8]) may be unreliable due to irregular conditions of the terrain.

Since obtaining an accurate metric map of the environment may not be possible in these scenarios, we propose an approach for constructing a map that captures robust topological information and approximate geometric information of the environment. This map is built from coordinate-free local encounters between the agents, which are merely binary observations of other agents in range, and do not include any range or other metric information. We refer to this representation as a Geometric Topological Persistent Map (GTP - Map) of the environment. Such maps can be used for identifying local and global homology (i.e., connected regions and holes) of the space, identify homotopic trajectories connecting regions in the space, and provide an approximate and intuitive visualization of the space. Additionally, the topological information can be used as a constraint to ensure that a metric reconstruction properly captures connectivity in the space as it is done via loop-closing in traditional SLAM approaches. Moreover, instead of providing continuous control feedback to the agents, we exploit natural stochastic motion models of the biobots and their encounter information. These strategies for motion and sensing accommodate for the hardware limitations of the platforms under consideration.

In this paper, we extend the methodology introduced in [9] for mapping of unknown environments under the constraint of limited sensing information using bio-inspired agents, where a notion of encounter metric was built based on local interactions among the agents. We exploited tools from topological data analysis [10] to extract spatial information such as connected components and holes (referred to as topological features) of the environment and build a map of persistent and robust topological features. We implemented the approach in [9] on a robotic platform, the WolfBot [11]. Although the methodology in [9] works quite well for simple scenarios [12], its performance and accuracy in topological feature classification degrades for more complex and larger environments with increasing number of persistent features in the space. Therefore, we extend the original approach by proposing a dynamic mapping strategy which conducts exploration and map construction at local and global levels via the use of an aerial leading agent (e.g. a UAV as shown in Figure 1). At a global level, the aerial agent performs a dynamic sweeping coverage of the area of interest by leading the biobotic swarm. Information gathered over small time windows are exploited to build local maps which are then stitched together in order to build a global map (see Figure 2).

The remainder of the paper is organized as follows: An overview of the related work to this study is provided in section 2 . Section 3 describes the problem under study including models and assumptions for biobotic agents as well as the UAV, followed by a more detailed modeling of a biobotic swarm in section 4. A concise background on the tools from topological data analysis used in this work is provided in section 5 . We present an overview of our mapping methodology in section 6. Metric estimation for point cloud construction is described in section 7 , followed by topological data analysis in section 8. Map merging for extracting global maps out of local maps is discussed in section 9, and section 10 illustrates our approach via numerical simulations and experiments with a swarm of Hexbugs [13]. Finally, discussion and conclusions are provided in sections 11 and 12, respectively. 


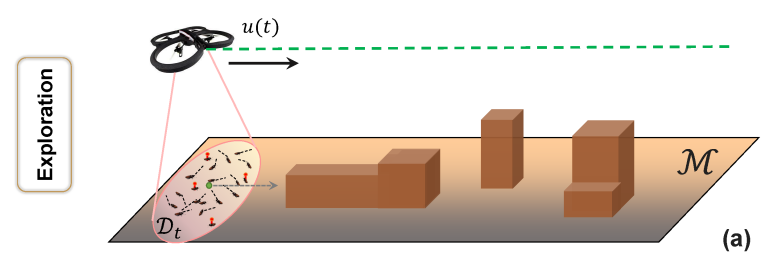

(a)

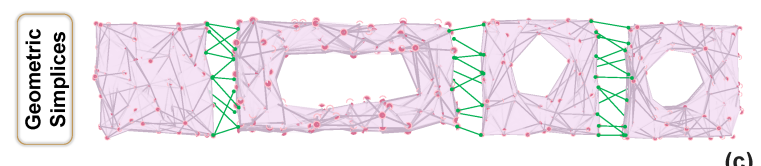

(c)

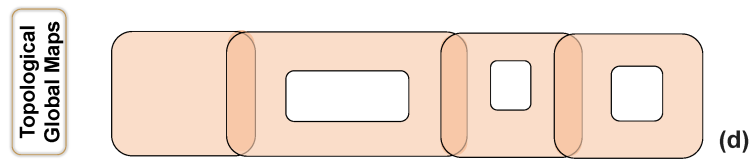

Figure 2: An overview of our mapping approach: (a) An example of a physical domain $\mathcal{M}$ to be explored with the swarm of agents, where $\mathcal{M}$ is the area of interest and $\mathcal{D}_{t}$ denotes the domain covered by the aerial agent at time $t,(\mathrm{~b})$ sliding time windows for local map construction, (c) desired geometric simplicial maps for each window (in pink) stitched with green edges, (d) desired global topological map after merging.

\section{Related Work}

Simultaneous localization and mapping (SLAM) techniques estimate the position of moving agents based on a set of observations from landmarks in the environment as well as sensing information from the moving agents (e.g., odometry, IMU, or vision) [14]. The majority of recent approaches assume that the exploring agents are equipped with highbandwidth sensing devices such as cameras or depth sensors [5, 15]. In this paper, we focus on minimal sensing strategies for robotic swarms in which such sensing modalities are unavailable.

Related to our work is the research on cooperative mapping with robotic networks. Recent advances in graph based approaches have made remarkable progress in the cooperative mapping results [16]. However, most cooperative approaches require huge computational resources and memory as a result of using visual sensing [17]. Cooperative SLAM on small mobile robots with limited sensing and computational resources have been studied in [18]. The robots however are equipped with laser pointers and vision sensors in order to compute dis- tances. Leung et al.[19] have studied decentralized coordination and estimation for multi-robot SLAM for sparsely communicating robots in a dynamic network, where robots share their history of local maps over time as they get disconnected. Benedettelli et al.[20] developed a multi robot mapping technique using the recent $\mathrm{M}$-space representation which merges maps across pairs of robots whenever they meet. The robots however move according to predefined trajectories, whereas in our work their motion is a stochastic process. Furthermore, each robot creates its own local map, and maps are merged when two robots meet, whereas in our work, local maps are also constructed cooperatively. In [21], exploration and coordination strategies for multi-robot mapping are studied. In this work, we leave the exploration to a great extent to natural exploration capabilities of the biobotic insects. Map merging under communication constrains in robotic networks using consensus is addressed in [22]. The communication constrained multi-robot SLAM approach of [23] employs a notion of encounters for sharing pairwise measurements between agents similar to our work but is particularly designed for underwater vehicle applications.

During the past decade, researchers in experimental biology and biomedical engineering have investigated models for the motion of individual cockroaches as well as groups of them based on experimental data [24]. Hardware platform developments include a matched size miniature robot simulating the individual and group behavior of cockroaches based on hierarchical potential fields [25], and a small autonomous robot equipped with an onboard camera and antenna-like contact sensors [26]. Development of insect biobots which can be remotely controlled through different type of stimulants has attracted a lot of attention in recent years. Cao et al. [27] recently have demonstrated remotely controlled beetle biobots by applying electrical stimulants to their leg muscles [28], and control the insects step length and walking speed by varying the duration of muscular stimulation signal. $\mathrm{Li}$ and Zhang [29] have reported motion control of cyborg cockroaches via human brain by recognizing human motion intention and sending appropriate pulse trains to the antenna nerve to stimulate their brain. In [30] a hybrid biobotic system consisting of American and discoid cockroaches has been developed which employs an alternative locomotion control by applying the signals to the nervous system. Schwefel et al.[31] study wireless communication by 
self-powered discoid cockroach biobots by implanting biofuel cells to convert insects' chemical energy into electrical signals. To our best knowledge, all of these studies have considered individual control of biobots, and one has made progress towards the development of frameworks for exploiting biobotic systems for robotics tasks such as localization and mapping.

Methods from computational topology can provide tools to extract topological features from data sets without requiring coordinate information. This makes them more suitable for scenarios in which weak or no localization is provided. Topological data analysis (TDA) [10], is a new approach to data analysis which employs tools from persistent homology theory [32] to obtain a qualitative description of the topological attributes and visualization of data sets sampled from high dimensional point clouds.

TDA recently has been successfully applied in a variety of applications including image processing and computer vision [33], gene expression data [34] dynamical systems [35], target tracking [36], signal processing [37], and activity recognition [38]. Topological frameworks have been also employed on applications in sensor networks and robotics. Particularly, these methods have been used for coverage and hole detection in sensor networks [39, 40], motion planning [41], and localization [42, 43]. However, these studies focused on stationary networks, and are mainly concerned about the coverage holes in the sensing domain of the network rather than the characterization of the physical environment itself. Ramaithitima et al. [44] consider the coverage of unknown environment under noisy and limited sensing information and no metric or localization information. Although this work is closely related to ours in terms of the sensing and localization constraints and mathematical tools, it differs in the sense that it focuses on sensor deployment rather than mapping. Moreover, by considering a purely topological algorithm, they bypass the metric requirement while we try to estimate a metric and build our topological framework on top of the obtained metric space.

Walker [45] employed persistent homology to compute topological invariants from encounter data of the mobile nodes in mobile ad hoc networks in order to infer global information regarding the topology of a physical environment, but the nodes are assumed to follow a simple mobility model on a graph. A topological version of localization via signals of opportunity has been studied in [43]. However, this approach is mostly focused on the localization problem although it could also detect coarse features of the environment in the context of mapping. Furthermore, it requires the existence of transmitters in the environment that provide such signals of opportunity. Topological localization and mapping has been also considered lately in [42] and [46]. Although these are akin in the context of using topology for representation of physical structures in the environment with mobile agents, they differ from our work in the sense that they assume the existence of an IMU or a motion model for prediction of the agents' states, as well as fixed landmarks on the boundaries of structures (e.g. buildings) to be used for observation. In this paper, however, the mapping is intended to be performed using local encounters among the agents and not relying on external resources (signals of opportunity or known fixed landmarks), as they may be unavailable as a consequence of the emergency response scenarios under consideration.

There exists also a notion of topological SLAM in the literature (e.g. [47, 48]), which differs from our work in the sense that the constructed topological maps in these studies do not contain any metric nor positional information, and only concern about the connectivity of the free space of the environment, represented as connectivity graphs.

\section{Problem Description}

Consider a collection of mobile agents moving stochastically in a bounded domain of interest $\mathcal{M} \subset$ $\mathbb{R}^{2}$ on the ground. We restrict our analysis to a 2D environment for simplicity while the techniques introduced do not rely on this assumption. Each ground agent is distinguished by its unique ID (e.g. MAC address), and its motion dynamics in the bounded space mimics the movement model of a biobotic insect. The movement model of the biobotic agents on the ground can be described by two types of motions: natural motion and controlled motion, which will be described in section 4 .

An aerial leading UAV (L-UAV) (e.g. a quadcopter or a biobotic moth [49]) plays the role of a swarm leader in order to herd the ground agents to explore all parts of the desired environment over time. It also serves as an access point to establish a local network for communication with the swarm in order to send control commands and receive required information (Figure 2). Alternatively, due to weak transmitters on the ground agents, we 
(a)

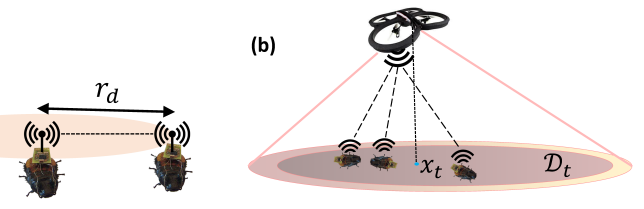

(c)

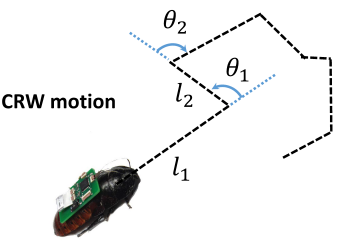

Figure 3: Biobotic swarm modeling:(a) Encounter region for two biobots, (b) communication visibility range and coverage domain of the UAV and (c) the correlated random walk (CRW) model for the biobots

could consider an ad-hoc communication network between the biobots.

The ground agents are provided with weak localization information. Specifically, binary local interactions among the agents when they are within a distance of $r_{d}$ of each other (see Figure 3(a)), is the only piece of information provided. No coordinates or other localization information is available. Note that odometry and inertial measurements are considered to be too unreliable due to the uneven and unstructured terrains present in our application scenario.

It is assumed that the leader is moving in a horizontal 2D plane at a constant linear speed of $v_{L}$ while maintaining a constant height with respect to the ground. Algorithms for control and stability of aerial vehicles are well studied in the literature (e.g. $[50,51])$; hence, we do not focus on the control strategies for the UAV. Furthermore, we define a bounded coverage domain $\mathcal{D}_{t} \subset \mathcal{M}$ at time $t$ around the UAV in the azimuth plane which specifies a neighborhood around the L-UAV based on signal strength from its wireless transmitter [52]. A simple model for this region is a circular disk with fixed radius $r$ at a specific height (see Figure 3(b)). The coverage domain will ensure appropriate communication links and will be used to specify a virtual fence for herding the biobots.

The problem of sweeping an area in order to achieve coverage of the whole domain (dynamic or sweeping area coverage) has been extensively studied in the literature (e.g. [53]). Hence, we assume that the L-UAV is performing a sweeping coverage with simple strategies such as following straight line trajectories or back and forth motion sweeping.

Given this minimal sensing scenario, our goal is to construct a map of the unknown environment while efficiently exploring the domain. As the swarm of agents explore the environment, a map of the scene is built dynamically by having the agents share information about their local interactions referred to as encounters. This map consists of geometric point clouds representing estimates of distances between the sampled points, and a summary of the environment in terms of topological features (e.g. connected components and obstacles). We refer to such a map as Geometric Topological Persistent Map (GTP - Map) of the environment.

\section{Biobotic Swarm Modeling}

Models for motion, sensing and communication of the biobotic agents are introduced in this section.

\subsection{Motion Model}

For the biobotic agents on the ground, we assume two types of motions: natural motion and controlled motion.

Natural Motion (NM). The natural motion model of the ground agents is adopted from the statistical motion model of individual cockroaches in bounded spaces in [54], described for completeness in this section. The individual movement of cockroaches can be described mainly by two behaviors, namely correlated random walk (CRW) [55] (or diffusive random walk), and wall following (WF). Cockroaches, in their natural mode, move according to a CRW model when they are far enough from the boundaries of the domain, and perform WF behavior when they detect the edges of the environment using their antennas [54]. During WF, they switch back again to CRW motion towards the interior of the domain after some stochastic amount of time. Moreover, it is known that during their CRW or WF motion, the insects probabilistically stop for some period of time and then continue their movement [54].

The CRW is modeled as piecewise linear movements with fixed orientation, characterized by line segments $\left\{l_{i}\right\}$ interrupted by changes in direction $\left\{\theta_{i}\right\}$, and constant average velocity of $v_{m}[54,55]$. The lengths of line segments $l$ has an exponential distribution with characteristic length $l^{*}$ :

$$
p(l)=C e^{-l / l^{*}},
$$


where $C$ is a constant. Changes in orientation is considered to be isotropic, where $p\left(\theta_{i+1} \mid \theta_{i}\right)=$ $p\left(\theta_{i+1}\right)$ with a uniform distribution $U[0,2 \pi)$. Changes in direction are also triggered by collision detection with other agents or obstacles in the scene. A typical velocity for the biobots in a search and rescue mission can be considered between 0.05 to $0.5 \mathrm{~m} / \mathrm{s}$. The agents' natural stopping behavior is also described by a stochastic process which occurs during the motion with a probability of $p_{\text {stop }}$, and the length of stop periods is described with multi-modal exponential distributions. We refer to the insects being in a static $(S)$ mode when they stop. Figure 3(c) shows an example of CRW motion with few line segments $l_{i}$, with angular reorientations of $\theta_{i}$.

Cockroaches also manifest a group behavior, $a g$ gregation, when they interact with each other [24]. In this paper, however, we focus on the CRW behavior for simplicity. Dirafzoon et al. in [9] explored the effect of WF behavior on the exploration and mapping process.

Hence our model of natural motion will be reduced to a two state graphical model switching between CRW and S, which can be considered as a natural mode of operation for biobotic insects [2]. Note that although this model is particularly developed and studied for German cockroach Blattella germanica, many animals (in particular other species of cockroaches) are known to display similar motion behaviors with possibly different types of CRW or Levy Walk [55].

Controlled Motion (CM). The remotely controlled biobotic insects are equipped with system-on-chip based backpacks enabling a controlled motion mode by responding to commands of the following types: turn left, turn right, start motion or stop motion. These commands are employed in order to: (1) control the stop times of the agents, and (2) control the swarm to stay within the coverage domain of the LUAV by enforcing a virtual fence relying purely on signal strength. The implementation of a virtual fence for biobots using visual feedback has been demonstrated in [3]. For the scenarios in which exact localization is not available, it is possible to develop algorithms for navigation that make use of wireless signal strength [56], which may incorporate stochastic strategies such as those performed by bacteria through chemotaxis [57].

\subsection{Proximity Sensing and Communication}

The proximity sensing and communication model for the ground agents is inspired by limited sensing capabilities of the biobotic insects, consisting of a wireless transmitter and receiver provided by a system-on-chip based ZigBee enabled wireless backpack system attached to their bodies [2]. Each agent is assumed to have a limited proximity sensing capability, and can identify and communicate with other agents within a detection radius $r_{d}$, as shown in Figure 3(b). For a typical search and rescue application $r_{d}$ can be set to an order of $1 \mathrm{~m}$. The agents are able to record their encounters with each other in accordance with the corresponding times as encounter events. These encounters are in fact the main piece of information in the construction of a metric on the samples of the physical space for the purpose of mapping. Furthermore, the nodes are able to report their status as being in a CRW or static state. Note that we do not consider any external obstacle detection/collision mechanism as we rely on insects' "built-in" instinct for collision detection and their natural change of direction in case of collision; hence, we do not require any additional sensors for obstacle detection/avoidance.

\section{Background on Topological Data Analysis}

In this section, we present a brief introduction of some of the basic concepts in topological data analysis (TDA) used throughout this paper. A comprehensive review of the topic can be found in [10].

Topological data analysis employs algorithms from computational topology to represent the prominence of features in a point cloud in terms of a compact representation of the multi-scale topological structure called persistence diagrams [10]. Consider a manifold $\mathcal{M}$ whose structure and features are of our interest (e.g. an unknown environment to be mapped). The space $\mathcal{M}$ usually is not directly accessible but a sampled version of it, $\mathcal{X}=\left\{x_{1}, \ldots, x_{n}\right\}$, can be used for computations. This sample is represented as a point cloud, a finite set of points equipped with a metric, defined by pairwise distances between the points (distance function). The distance function can be obtained directly using the coordinates of the points (if available), or can be constructed by estimation of pairwise distances independently from their (possibly unknown) coordinates. 
(a)

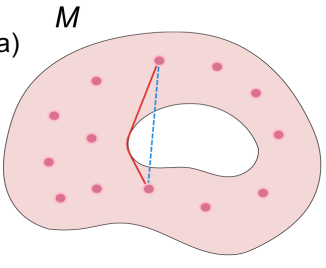

X

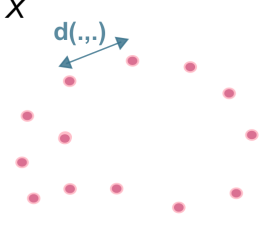

(b)

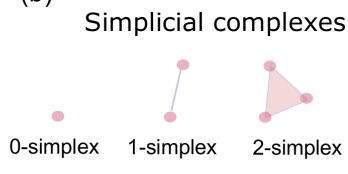

(d)

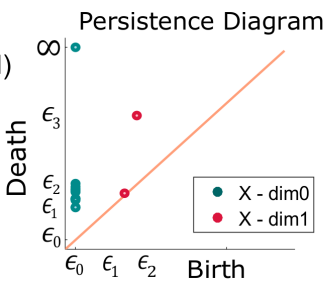

(c) Filtration
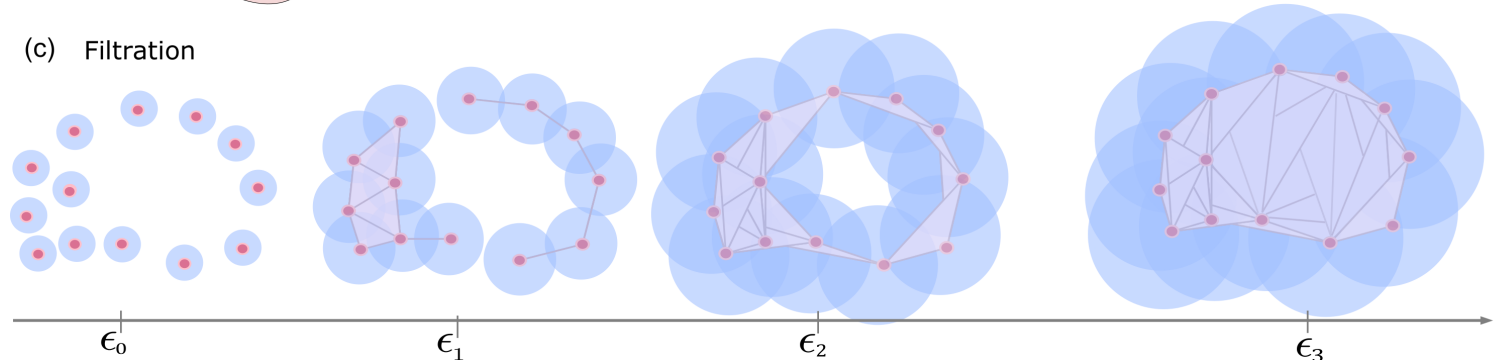

Figure 4: Topological persistence: (a) An example of a space $\mathcal{M}$ with a sampled point cloud $X$, (b) illustration of simplicial complexes, (c) a filtration of simplicial complexes over $X,(\mathrm{~d})$ the corresponding persistence diagram with $\mathrm{PD}_{0}(X)$ in green and $\mathrm{PD}_{1}(X)$ in red, highlighting persistence of connected components and holes over the scale, respectively.

\subsection{Simplicial Complexes}

A standard method to analyze the topological structure of a point cloud is to map it into combinatorial objects called simplicial complexes. Given a set of vertices $\mathcal{X}$, a $k$-simplex is defined as a set

$$
\left\{x_{1}, x_{2}, \ldots, x_{k+1}\right\}, x_{i} \in \mathcal{X}, \forall i \text { and } i \neq j, \forall i, j \text {. }
$$

For example, a 0-simplex is a vertex, a 1-simplex is an edge, and a 2-simplex is a triangle. A finite collection of simplices is called a simplicial complex if for each simplex each of its faces are also included in the collection.

Figure 4(b) shows an example of 0, 1, 2-simplices constructed on points drawn from $\mathcal{M}$. One way to build these complexes is to select a scale $\epsilon$, place balls of radius $\epsilon$ on each vertex, and construct simplices based on their pairwise distance relative to $\epsilon$. A computationally efficient complex, called the Vietoris-Rips complex $\mathcal{R}(\mathcal{X}, \epsilon)$ [58], consists of simplices for which the distances between each pair of its vertices are at most $\epsilon$. In other words, any subset $\mathcal{X}^{\prime} \subset \mathcal{X}$ is a simplex in $\mathcal{R}(\mathcal{X}, \epsilon)$ if and only if

$$
d\left(x_{i}, x_{j}\right) \leq \epsilon, \forall x_{i}, x_{j} \in \mathcal{X}^{\prime} .
$$

\subsection{Persistent Homology}

Topological invariants of a space $\mathcal{M}$ are mappings which aim to classify equivalent topological objects into the same classes. Homology (a type of topological invariance) can be summarized as a compact representation in the form of so-called Betti numbers, which are the ranks of the homology groups. The $n$-th Betti number, $\beta_{n}$ is a measure of the number of $n$-dimensional cycles in the space (e.g. $\beta_{0}$ is the number of connected components and $\beta_{1}$ is the number of holes in the space). For a dense enough sample, one can find an interval for the values of $\epsilon$, for which constructed simplicial complexes belong to the same class of topological invariants as the space $\mathcal{M}$. However, finding the proper values for $\epsilon$ is a strenuous job.

On the other hand, persistent homology theory considers construction of a sequence of such complexes for the sampled point cloud over multiple scales $\epsilon$, called a filtration, and extracts geometrical and topological features of the dataset at specific scales. A filtration $X(\epsilon)$ can be obtained by increasing $\epsilon$ over a range of interest, with the property that if $t<s$, then $X(t) \subset X(s)$. Persistent homology computes the values of $\epsilon$ for which the classes of topological features appear $\left(b_{n}^{i}\right)$ and disappear $\left(d_{n}^{i}\right)$ during filtration, referred to as the birth and death values of the $i$-th class of features in dimension $n$. This information is encoded into persistence intervals $\left[b_{n}^{i}, d_{n}^{i}\right]$, or by a compact representation called persistence diagram [59]. The persistence diagram $\mathrm{PD}_{n}(\mathcal{X})$ is a multi-set of points $\left(b_{n}^{i}, d_{n}^{i}\right)$ in the extended plane that summarizes how topological features of the point cloud vary over the scale $\epsilon$. Algorithms for computation of persistent homology can be found in [60]. 


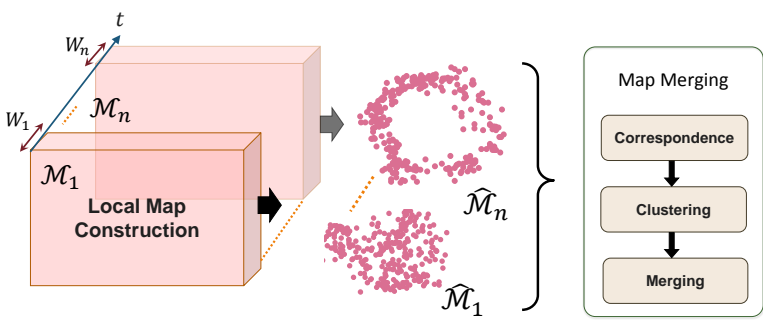

Figure 5: GTP Mapping: A block diagram illustrating construction of local maps over sliding time windows (left), example generated local point cloud maps $\hat{\mathcal{M}}_{i}$ (middle), and the components for map merging of local maps $\hat{\mathcal{M}}_{i}$ from each window. The pipeline for construction of local maps is illustrated in figure 6 .

An example of a topological space $\mathcal{M}$ together with sampled data $\mathcal{X}$ and corresponding filtration over $\epsilon$ is shown in Figure 4. In the persistence diagram in Figure 4(d), features corresponding to dimension 0 are shown in green and the ones corresponding to dimension 1 in red color. The space $\mathcal{M}$ can be described topologically as having 1 connected component and 1 hole, meaning that $\beta_{0}=1$ and $\beta_{1}=1$ for this example. Although there exist several topological features in the PD, only 2 of them can be distinguished from the rest based on their distant from the diagonal. These points correspond to the persistent features with longer persistent intervals, referred to as topological signals, while the rest are short-lived features that are referred to as topological noise [61]. Hence one can infer the existence of one connected component and one persistent hole from the PD.

\section{The Proposed Approach}

In this section, we present an overview of our proposed algorithmic approach for GTP-Mapping. Mapping is performed dynamically by exploiting the natural stochastic behavior of insects augmented with their controlled mode for coordination with the L-UAV. The agents' interactions are used locally to construct a local GTP-Map of the domain $\mathcal{D}_{t}$, while the L-UAV is responsible for guiding the swarm over the entire domain. Local maps are stitched together in order to construct a global GTP-Map. The process consists of the following steps: (1) agent dispersion over the space, (2) selection of static agents as landmarks, (3) gathering of encounter events, (4) construction of local GTPMaps, and (5) construction of a global GTP-Map.

\subsection{Agent Dispersion}

The local exploration is realized by dispersion of the ground agents throughout the local domain by performing CRW motion, which has been shown to be an effective method for dispersion of swarms in bounded environments [62]. During this initial stage, the L-UAV hovers over a fixed position. After the initial dispersion stage, the L-UAV starts sweeping the environment according to the description of section 3 in order to attain dynamic coverage of the environment. Agents remain within the coverage domain of the L-UAV by using the controlled motion strategies described in section 4 .

\subsection{Static Landmark Selection}

At any given time point, a subset of agents in the coverage domain are set to static mode. Denote by $\mathcal{S}(t)$ the set of static nodes at time $t$. These nodes will play the role of landmarks in the mapping algorithm, and their stationary unknown position will be used to compensate for drifts in the metric estimation, which will be illustrated in section 7 . The landmark selection process identifies a subset of nodes by a modified version of standard Maxmin landmark selection algorithm [63]. Given the adjacency graph due to the communication range, we use its hop distance to select static agents iteratively by maximizing the distance of the nodes from the previous set of static agents. The first agent is selected randomly. If the graph is not connected, we assume that static agents are selected randomly from each cluster with probability equal to the percentage of agents in each cluster. If any of the static agents leaves $\mathcal{D}_{t}$, it immediately switches to CRW behavior, and a new static agent is selected.

Note that the notion of landmarks here is different from the one in the witness complex methodology of [9]. The landmarks in the witness complex are subsampled points from the point cloud with an attempt to reduce its size while preserving its topological features, while here landmarks are stationary nodes for map correction. The former notion of landmark set is referred to as the subsampled point cloud in this paper. 


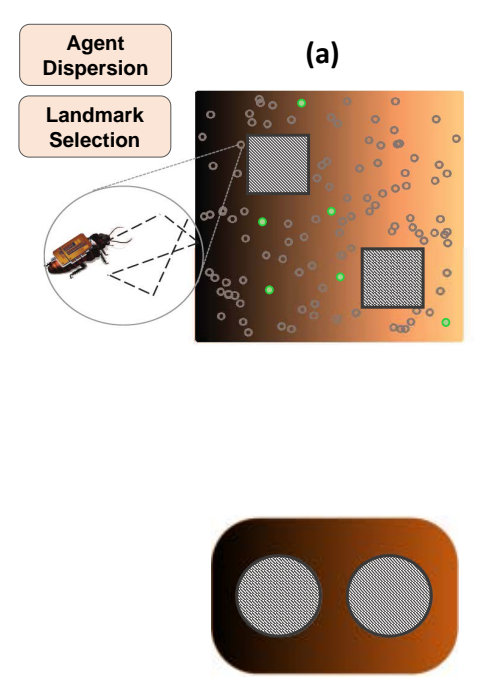

(f)

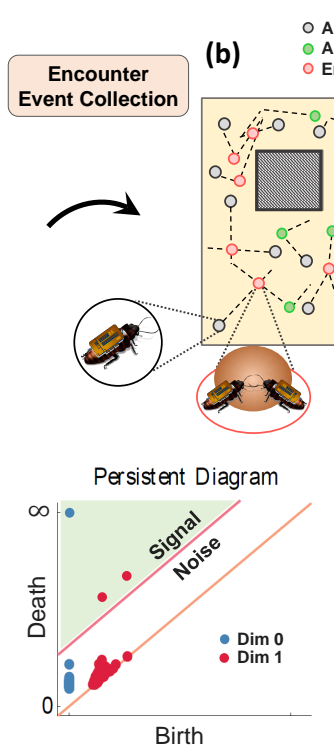

(e)
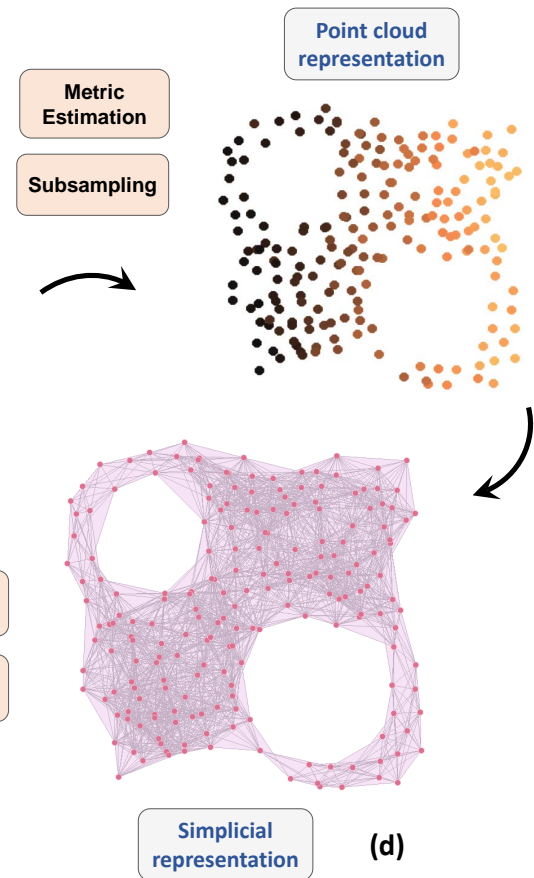

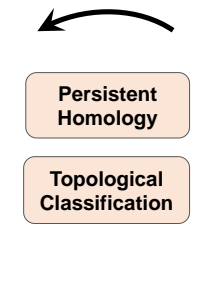

presentation

Figure 6: Simulation results illustrating the pipeline for construction of a local GTP-Map: (a) a square shape physical environment with two obstacles and a snapshot of the location of 100 moving biobots shown as gray circles and static landmarks (green dots) (b) a subsample of collected coordinate-free encounter nodes over which a metric is estimated (shown as pink circles), (c) the point cloud obtained from subsampling the projection of the estimated encounter metric, (d) a simplicial Rips-complex representation, (e) persistence diagram of the complex highlighting connected components in green and holes in red dots classified as signals and noisy features, and (f) a topological sketch of the environment. Different components of the pipeline are described in details in sections 6,7 and 8.

\subsection{Encounter Event Collection}

Whenever there is an interaction between agents (static or moving), an event is recorded including the IDs of the agents involved, the corresponding time, as well as the state (static or moving) of the agents. This information is assumed to be sent to the L-UAV in a centralized version of our approach, or passed along to neighboring agents in a distributed implementation. Note that this will be the only piece of information used in mapping and no coordinate information will be acquired.

\subsection{GTP-Map Construction}

As the leading agent sweeps the environment, observations over a set of intersecting time windows are used to construct local GTP-Maps. Information from observations in the intersection of these windows are used to determine correspondence between these maps in order to stitch them together and build a global GTP-Map. An overview of the approach is shown in Figure 5.
The overlapping time windows considered of the form:

$$
W_{i}=\left[t_{i-1}-\Delta t / 2, t_{i}+\Delta t / 2\right], i=1, \ldots, N_{w},
$$

where $\left\{t_{i}\right\}_{i=1}^{n}$ are evenly spaced, $N_{w}$ is the total number of windows, and $\Delta t$ is the amount of overlap (see Figure 2 for an illustration). Note that $\Delta t$ needs to be large enough in order to guarantee that enough landmarks and encounter events exist within the corresponding spatial domain of the overlapping window so that the local maps are properly stitched. The local domain corresponding to the $i$-th time window is specified by

$$
\mathcal{M}_{i}=\mathcal{M} \cap \bigcup_{t \in W_{i}} \mathcal{D}_{t} .
$$

Local GTP-Maps for the domains $\mathcal{M}_{i}$ are constructed using a modified version of the approach introduced in [9]. Our modified methodology consists of a refined estimation of a metric over the set of encounters exploiting the stationary nodes as 
landmarks (see metric estimation in section 7), and the extraction of robust topological features from density based subsampled landmarks and classification (see topological estimation and classification in section 8). Figure 6 illustrates the procedure for constructing a local GTP-Map through simulation results obtained for a squareshape physical environment including two obstacles. Stitching of the local maps to obtain a global map is addressed in section 9 .

\section{Metric Estimation}

An encounter metric is generated in order to estimate the pairwise distances over the set of encounter events. This metric is an approximation of the manifold distance between the sample points in the space $\mathcal{M}$.

An encounter event takes place between two moving agents (or a moving and a static agent) if their corresponding center positions fall within a distance of $r_{d}$, i.e. $\left\|p_{i}-p_{j}\right\| \leq r_{d}$. (see Figure 3 ). An encounter $\mathbf{e}_{i}$ is recorded by an agent as a tuple:

$$
\mathbf{e}_{i}=\left[t_{i}^{1}, t_{i}^{2}, \mathrm{ID}_{i}^{1}, \mathrm{ID}_{i}^{2}\right]
$$

where $\left[t_{i}^{1}, t_{i}^{2}\right]$ denotes the time interval of the encounter, and $\mathrm{ID}_{i}^{1}$ and $\mathrm{ID}_{i}^{2}$ represent the IDs of the two encountering agents. Define $T\left(\mathbf{e}_{i}\right)=\left[t_{i}^{1}, t_{i}^{2}\right]$ as the $i$-th event time interval, and $\operatorname{ID}\left(\mathbf{e}_{i}\right)=$ $\left\{\mathrm{ID}_{i}^{1}, \mathrm{ID}_{i}^{2}\right\}$ as the ID set of the event $\mathbf{e}_{i}$. and denote the set of all events as $\mathcal{E}=\left\{\mathbf{e}_{i}\right\}_{i}$. Assigned to each event $\mathbf{e}_{i}$ is a coordinate $q_{i}=\left[p_{i}, t_{i}\right] \in \tilde{\mathcal{S}}$, where $p_{i} \in \mathcal{S}$ represents the center of mass for the unknown set of locations at which $\mathbf{e}_{i}$ occurs, and $t_{i}$ represents the encounter time interval center as $t_{i}=\left(t_{i}^{1}+t_{i}^{2}\right) / 2$. Furthermore, define the landmark set $\mathcal{L}=\left\{l_{k}\right\} \subset \mathcal{I}$ as the set of IDs of the agents that are assigned to be static for some period of time.

Given the set $\mathcal{L}$ then the set of landmark events corresponding to the $k$-th landmark is defined as

$$
S_{k}=\left\{\mathbf{e} \mid l_{k} \in \operatorname{ID}(\mathbf{e})\right\} .
$$

Consider the set $\mathcal{E}_{k} \subset \mathcal{E}$ of unlocalized samples gathered from the encounters in a local domain $\mathcal{M}_{k}$. We construct an undirected weighted graph $\mathcal{G}$ over $\mathcal{E}_{k}$ with vertices corresponding to the events $\mathbf{e}_{i}$, denoted as the encounter graph. Given graph $\mathcal{G}$, for any two vertices $\mathbf{e}_{i}$ and $\mathbf{e}_{j}$, include an edge $e_{i j}$ if they have a common agent involved in both events, i.e. $\operatorname{ID}\left(\mathbf{e}_{i}\right) \cap \operatorname{ID}\left(\mathbf{e}_{j}\right) \neq \emptyset$. The corresponding weight $w_{i j}$ will represent pairwise distances which are calculated as follows. For $\mathbf{e}_{i}$ and $\mathbf{e}_{j}$ define the pairwise time metric $d_{\mathcal{T}}\left(\mathbf{e}_{i}, \mathbf{e}_{j}\right)$ as:

$$
d_{\mathcal{T}}\left(\mathbf{e}_{i}, \mathbf{e}_{j}\right)= \begin{cases}\left|t_{i}-t_{j}\right|, & \text { if } \operatorname{ID}\left(\mathbf{e}_{i}\right) \cap \operatorname{ID}\left(\mathbf{e}_{j}\right) \neq \emptyset \\ \infty, & \text { otherwise }\end{cases}
$$

Note that, for connected nodes $\mathbf{e}_{i}$ and $\mathbf{e}_{j}$, the Euclidean distance between the corresponding coordinates $p_{i}$ and $p_{j}$ is upper bounded as:

$$
\left\|p_{i}-p_{j}\right\| \leq v_{m} \cdot\left|t_{i}-t_{j}\right|
$$

Provided that the agents were moving only on straight lines and there were no obstacles in the field, this upper bound could have precisely approximated the Euclidean distance. However, due to the random walk motion of the agents, the accumulated errors of the pairwise distances over time will cause large drifts in metric estimation.

In order to reduce the amount of such drifts, we furthermore improve our graphical model by incorporating the fact that landmark nodes are static. Consider again the set $\mathcal{L}$ of the landmark nodes. For each $l \in \mathcal{L}$ let

$$
\mathcal{T}(l)=\bigcup_{i} T^{i}(l)
$$

denote the union of all time intervals $T^{i}(l)=$ $\left[t_{l}^{i, s}, t_{l}^{i, f}\right]$ for which agent $l$ has been in a static mode, where $t_{l}^{i, s}$ and $t_{l}^{i, f}$ correspond to the start and end times of each interval $T^{i}(l)$, respectively.

Then, for two events $\mathbf{e}_{i}$ and $\mathbf{e}_{j}$, if there exists a landmark event cluster such that

$$
I D\left(\mathbf{e}_{i}\right) \cap I D\left(\mathbf{e}_{j}\right)=l \in \mathcal{L},
$$

they had to occur geometrically at nearby locations if the landmark node has not moved within the time interval of two encounters, i.e.

$$
T\left(\mathbf{e}_{i}\right) \cap T\left(\mathbf{e}_{j}\right) \cap \mathcal{T}(l) \neq \emptyset .
$$

Therefore, the corresponding weight $w_{i j}$ is set to 0 in this case, and we define the pairwise encounter metric $d_{\mathcal{E}}(.,$.$) as$

$$
d_{\mathcal{E}}\left(\mathbf{e}_{i}, \mathbf{e}_{j}\right)= \begin{cases}0 & \text { if (11) and (12) hold } \\ d_{\mathcal{T}}\left(\mathbf{e}_{i}, \mathbf{e}_{j}\right), & \text { otherwise }\end{cases}
$$


To proceed with the construction of geometric point cloud, we perform a graph shortest path algorithm on the graph $\mathcal{G}$, and define our encounter graph metric on the nodes in $\mathcal{G}$ as:

$$
d_{\mathcal{G}}^{\mathcal{E}}\left(\mathbf{e}_{i}, \mathbf{e}_{j}\right)=\min _{\mathcal{P}}\left\{d_{\mathcal{E}}\left(x_{0}, x_{1}\right)+\ldots+d_{\mathcal{E}}\left(x_{p-1}, x_{p}\right)\right\},
$$

where $\mathcal{P}=\left(x_{0}=\mathbf{e}_{i}, \ldots, x_{p}=\mathbf{e}_{j}\right)$ denotes a path along the edges of graph $\mathcal{G}$ connecting $\mathbf{e}_{i}$ to $\mathbf{e}_{j}$. The shortest paths and their corresponding lengths can be found from Dijkstra's or Floyd's shortest path algorithms [64].

Graph $\mathcal{G}$ with edge weights set to the above metric, i.e. $w_{i j}=d_{\mathcal{G}}^{\mathcal{E}}\left(\mathbf{e}_{i}, \mathbf{e}_{j}\right)$, is referred to as the $E n$ counter Metric Graph. This metric approximates the geodesic distances over $\mathcal{M}$ of the sample points. We refer to the set of encounter nodes together with the corresponding estimated metric as the local $e n$ counter point cloud of the region $\mathcal{M}_{i}$, denoted as $\hat{\mathcal{M}}_{i}$. The estimated metric produces point clouds that are lying in higher dimensional spaces although their intrinsic geometry can be embedded in lower dimensional spaces. We make use of classical Multidimensional Scaling (MDS) [65] in order to find a $3 \mathrm{D}$ embedding of the point cloud data. MDS, is typically used for visualization of dissimilarity matrices by calculating a set of of coordinate whose configuration minimizes a loss function [65].

The procedure we acquire is closely related to the Isometric mapping (Isomap) technique [66] in manifold learning [67]. The main idea of Isomap is to estimate Geodesic distances on a manifold by the shortest path distance on the neighborhood graph of the samples taken from the manifold. However, the main differences here are that (1) in Isomap, the pairwise Euclidean distances between the neighboring nodes on a graph are known while we are dealing with a coordinate free system, and (2) the edge construction on the graph in Isomap is based on k-nearest neighbors or $\epsilon$-radius circles around each node, which again requires coordinate information.

Figure 6(c) shows the resulting point cloud obtained using the above procedure for a square local domain with total number of 100 agents with 10 of them being in $\mathrm{S}$ mode and the rest in CRW mode. One can clearly observe that although the point cloud is geometrically deformed with respect to the original space, it not only preserves topological features such as connected components and holes (obstacles), but also manifests some geometric features such as corners and the square-like shape of the environment to a great extent considering no coordinate information. This figure is color coded based on the true locations of the samples over $\mathcal{M}$, which is showing that the relative locations of obstacles are also preserved.

Metric Estimation Accuracy: The local metric estimation presented to obtain local point clouds of sub-domains can be viewed as the reconstruction of a manifold sampled from the space $\mathcal{D}_{i} \times$ $\left[t_{i-1}-\Delta t, t_{i}+\Delta t\right]$. Hence the accuracy of the procedure is highly dependent on the density of the samples taken from that space, which in turn depends on the number of agents, their average velocity, and the time assigned to explore a given subdomain. Moreover, the existence and appropriate density of the static landmarks used for correction of metric estimation errors, as well as connectivity of the constructed encounter graphs, play important roles in the construction of an accurate metric under weak localization conditions. We have investigated such dependencies to come up with bounds on the metric uncertainty and topological stability exploiting the existing literature on manifold learning [67] and topological inference and stability analysis [68]. In particular, in our recent work [12], we have been able to analytically examine the asymptotic behavior of the metric estimation and provide stability analysis for topological persistence. More specifically, we have shown that under certain conditions, our proposed metric estimates the geodesic distances in the underlying manifold with bounded errors. Under sufficiently large sampling density of the local regions in terms of encounter events, sufficiently large density of landmarks, and connectivity condition on the graph, it will converge to the geodesic metric. Quantitative analysis of metric convergence to the geodesic dissimilarities and their dependence on the number of moving agents and landmarks have been demonstrated through several experiments therein. Furthermore, the robustness of local map estimation with respect to the scaling and number of features has been investigated with random generation of obstacles in fixed size environment in [69].

Note that the type of uncertainties we are dealing with here is different from traditional SLAM approaches, where sensor measurements and pose uncertainties are of concern [70-72]. In our approach, which is a coordinate-free approach, however we consider edge weights corresponding to pairwise geodesic dissimilarities between samples, with uniformly bounded errors. As in traditional approaches for mapping, where probabilistic bounds 
on uncertainties are derived, one can make use of such bounds in our scenario as well by taking into the account the correlation between probabilistic motion model of the agents and the sampling density of their encounters. This is, however, beyond the scope of this work.

Consistency: Another important problem in mapping for large scale environments is related to the consistency $[70,71]$ of the map building process. Non-consistent results might be produced as a result of large accumulated uncertainties in large environments. There have been several approaches reported in the literature to evaluate whether the information in different parts of the map are consistent. For example, the information map in [70] is built upon probabilistic pose uncertainties in a lower rate than the original map, and its level sets are exploited as possible regions for exploration. The bounded metric uncertainties converging asymptotically as discussed above, provide an alternative characterization of consistency to the approaches based on probabilistic estimation. For example, uncertainties in the metric can be translated into confidence intervals for topological features as in [73]. Furthermore, one can assign probabilities to the edges on the encounter graph based on temporal information, with the assignment of larger weights to the edges connecting the nodes which are temporally closer. This yields to the construction of a probabilistic encounter graph and in turn a probabilistic metric estimation framework. Although developing similar consistency evaluation techniques for such a framework is plausible, it requires further investigation.

\section{Topological Estimation and Classification}

So far, we have constructed point clouds which provide geometric estimations for each local domain $\mathcal{M}_{i}$. However, such model is not precise and is subject to errors due to uncertainty on the distances, which may lead to deformations in the shape of the space. In order to address this issue, persistent homology provides a set of tools that are stable to bounded perturbations of the dissimilarity measure [68]. In this section, we present our methodology for extracting robust topological features from the point clouds, which introduces a statistical classification approach to separate noisy features from dominant ones. A high level overview of topological feature extraction is shown in Figure 6(d-f).
We process the encounter point clouds to construct a filtration of simplicial complexes, denoted as encounter complexes, over which persistent homology is computed to extract topological features in terms of persistence diagrams. This process is preceded by a subsampling step for cleaning up the data.

\subsection{Subsampling with Outlier Removal}

Extracting topological information from the whole point cloud would be computationally expensive due to the large number of events that are created over time, and it can be prone to error due to outliers on our estimated metric distance; hence a subsampling algorithm needs to be used to reduce the computational complexity and remove outliers while preserving the topological features of the point cloud. A common subsampling method in persistent homology which preserves topology is Maxmin algorithm [74], but it is very sensitive to outliers. Hence, we add a pre-processing layer for outlier removal using a nearest neighbor density estimation. Specifically, we employ $k$-nearest neighbors (KNN) filtering followed by a Maxmin subsampling algorithm to select a subset of points which preserves the topological information of the actual space. The KNN filtering is carried out by estimating the density of KNN's of each point and removing the ones whose KNN density is below a threshold of $q$-quantile of all values in the density map.

\subsection{Persistent Homology}

We use the mathematical tools introduced in section 5 in order to extract persistence diagrams from a filtration of the subsampled set of points from the filtered encounter point clouds constructed in the previous part. The filtration is based on computing the Vietoris-Rips complexes from the encounters as a function of the distance parameter $\epsilon$.

For computation of persistence intervals and persistent diagrams, we exploit the Dionysus $\mathrm{C}++$ library [75]. We restrict our computations to the dimensions 0 and 1 of persistence (corresponding to connected components and holes in the point cloud) as the higher dimensions are not applicable in our experiments.

\subsection{Topological Classification}

The previous analysis provides a methodology for extracting topological features from the point 
cloud. However, we also need to be able to separate persistent features from noisy detections due to subsampling and errors in metric estimation. Given a sampled point cloud $\mathcal{X}$ from a space $\mathcal{M}$, one constructs a filtration of simplicial complexes on $\mathcal{X}$ and analyzes variation in homology as a function of a threshold on the encounter metric. Nonetheless, persistence intervals do not provide a quantitative representation of the topology of the real space but only birth and death of the features as the value of $\epsilon$ varies. For a dense enough sampled data, however, one can find a range of values for which the homology of simplicial complexes is equal to the one for $\mathcal{M}$ [73]. Here, we propose a robust and scale-invariant classification algorithm for persistence intervals by learning such thresholds based on a non-parametric density estimation of noise in order to estimate the correct Betti numbers of the underlying space.

For our statistical analysis, let us define a collection of sampled point clouds $\left\{\mathcal{X}^{(p)}\right\}_{p=1}^{N}$ of a topological space $\mathcal{M}$. For each $\mathcal{X}^{(p)}$, we can extract from its persistence analysis a collection of $n$-th Betti number persistent interval lengths $\left\{l_{n}^{(p), k}\right\}_{k}$. Denote the $q_{n}$ quantile for this collection by $l_{q_{n}}^{(p)}$, and define the $n$-th estimated Betti number for the $p$-th point cloud as

$$
\hat{\beta}_{n}^{(p)}\left(\theta_{n}\right)=\sum_{k} \mathbb{1}_{\mathbb{R}^{+}}\left(\frac{l_{n}^{(p), k}}{l_{q_{n}}^{(p)}+\Delta_{n}}-\tau_{n}\right),
$$

where $\theta_{n}=\left(q_{n}, \Delta_{n}, \tau_{n}\right)$ is the estimation parameter vector, and $\mathbb{1}_{\mathbb{R}^{+}}$(.) is the indicator function of $\mathbb{R}^{+}$. This function specifies that only those persistent intervals for which the normalized value of $l_{n}^{(p), k}$ is greater than $\tau_{n}$ are considered as "true" persistent features. Furthermore, define the $n$-th Betti number error as

$$
e_{n}^{(p)}\left(\theta_{n}\right)=\left|\hat{\beta}_{n}^{(p)}\left(\theta_{n}\right)-\beta_{n}\right|
$$

where $\beta_{n}$ is the $n$-th Betti number of $\mathcal{M}$. Hence, the average error for the collection of sampled points can be expressed as

$$
e_{n}\left(\theta_{n}\right)=\frac{1}{N} \sum_{p=1}^{N} e_{n}^{(p)}\left(\theta_{n}\right)
$$

In order to perform classification with this metric, we generate a set of samples from the same topological space and train our classifier by finding the value of $\theta_{n}$ that minimizes the error function $e_{n}$. In order to make the cost function smooth for optimization purposes, one can replace the indicator function $\mathbb{1}(x)$ with a sigmoid function $\sigma_{\alpha}(x)=$ $\frac{1}{1+e^{-\alpha x}}$, where $\alpha$ is a scaling parameter. We denote by $\theta_{n}^{*}$ the optimal parameter value found using brute force optimization. Finally, we can compute the error $e_{n}\left(\theta_{n}^{*}\right)$ for a new collection of sampled data for testing purposes.

\section{Map Merging for Global Mapping}

This section presents a methodology to stitch the local maps for the domains $\left\{\mathcal{M}_{i}\right\}$ into a global map for $\mathcal{M}$ (see Figure 5). This problem, which is referred to as map merging in the mapping literature, is considered an essential part of mapping [76] and can be carried out in a variety of ways depending on the types of available sensory information (e.g. IMUs or visual sensing) or global localization information. As an example, in visual SLAM, frameto-frame registration of images is used to find the correspondence between partial maps [77].

The idea is to exploit the existence of common static landmark nodes between different maps to define a connectivity measure which is then used to merge the maps. To preserve topological features that cannot be described on a single local map (e.g. a very long structure that is not fully covered using a single observation window), we construct point clouds on the intersection of the consecutive map components and perform a clustering analysis to robustly identify the number of connections between the different map components.

\subsection{Correspondence}

Denote the intersection interval of the consecutive time windows $W_{i, i+1}=W_{i} \cap W_{i+1}$. We define the correspondence landmarks as a subset of static landmark nodes joining $\mathcal{M}_{i}$ to $\mathcal{M}_{i+1}$ as:

$$
\begin{aligned}
\mathcal{L}_{i, i+1}=\left\{l \in \mathcal{L}_{i, i+1}^{\prime}:\right. & \exists \mathbf{e} \in S_{l} \text { s.t. } \\
& \left.T(\mathbf{e}) \cap W_{i, i+1} \neq \emptyset\right\},
\end{aligned}
$$

where

$$
\mathcal{L}_{i, i+1}^{\prime}=\left\{l \in \mathcal{L}: \mathcal{T}(l) \subset W_{i, i+1}\right\} .
$$

$\mathcal{L}_{i, i+1}^{\prime}$ is the set of static nodes present throughout the window $W_{i, i+1}$. Condition (19) assures that the nodes in $\mathcal{L}_{i, i+1}^{\prime}$ have not moved within the period $W_{i, i+1}$. 
Furthermore, we define the set of correspondence encounters associated with these landmarks as

$$
\mathcal{E}_{i, i+1}=\left\{\mathbf{e} \in \mathcal{E}_{i} \cap \mathcal{E}_{i+1}: \operatorname{ID}(\mathbf{e}) \cap \mathcal{L}_{i, i+1} \neq \emptyset\right\} .
$$

An encounter metric is constructed on the set $\mathcal{E}_{i, i+1}$ in order to infer a metric on the set $\mathcal{L}_{i, i+1}$. In order to enrich $\mathcal{E}_{i, i+1}$ with more metric information from both point clouds $\hat{\mathcal{M}}_{i}$ and $\hat{\mathcal{M}}_{i+1}$, we extend it by including the KNN's of each event:

$$
\mathcal{E}_{i, i+1}^{\prime}=\mathcal{E}_{i, i+1} \cup\left\{\mathcal{N}_{k}(\mathbf{e}): \mathbf{e} \in \mathcal{E}_{i, i+1}\right\},
$$

where $\mathcal{N}_{k}(\mathbf{e})$ refers to the KNN's of e in the corresponding point cloud $\hat{\mathcal{M}}_{i}$ or $\hat{\mathcal{M}}_{i+1}$.

We construct the correspondence point cloud $\hat{\mathcal{M}}_{i, i+1}$ over the set of nodes $\mathcal{E}_{i, i+1}^{\prime}$ as follows:

1) Build a graph $\mathcal{G}_{i, i+1}$ over the set of nodes $\mathcal{E}_{i, i+1}^{\prime}$.

2) $\forall \mathbf{e}_{j}, \mathbf{e}_{k} \in \mathcal{E}_{i, i+1}^{\prime}$ set

$$
d_{\mathcal{G}}\left(\mathbf{e}_{j}, \mathbf{e}_{k}\right)=\min \left(d_{\mid \hat{\mathcal{M}}_{i}}\left(\mathbf{e}_{j}, \mathbf{e}_{k}\right), d_{\mid \hat{\mathcal{M}}_{i+1}}\left(\mathbf{e}_{j}, \mathbf{e}_{k}\right)\right)
$$

where $d_{\mid \hat{\mathcal{M}}_{i}}(.,$.$) denotes the induced metric from$ the point cloud $\hat{\mathcal{M}}_{i}$.

3) If $\operatorname{ID}\left(\mathbf{e}_{j}\right) \cap \operatorname{ID}\left(\mathbf{e}_{j}\right)=l \in \mathcal{L}_{i, i+1}$, set $d_{\mathcal{G}}\left(\mathbf{e}_{i}, \mathbf{e}_{j}\right)=0$.

4) Perform a graph shortest path algorithm on $\mathcal{G}$ and adjust the weights on edges accordingly.

The resulting metric on this point cloud, the correspondence metric, will have connectivity information to stitch edges of $\hat{\mathcal{M}}_{i}$ and $\hat{\mathcal{M}}_{i+1}$ together. Figure 7 shows an example of such stitching for two partial maps $\hat{\mathcal{M}}_{1}$ and $\hat{\mathcal{M}}_{2}$

Note that $\mathcal{L}_{i, i+1}$ corresponds to connections between the two maps, but some of these connections may correspond to the same connected component within the domains $\mathcal{D}_{i}$ and $\mathcal{D}_{i+1}$. In order to identify equivalence classes for the connections, we perform clustering over the correspondence encounter metric constructed on $\mathcal{E}_{i, i+1}$. As we do not know the number of clusters a priory, we employ the single linkage clustering as one of the most famous agglomerative hierarchical clustering techniques [78]. Hierarchical techniques provide a similarity dendrogram which can be used to classify data into clusters by applying a cut-off threshold $\tau$ on the similarity values. This cut-off is selected experimentally. Figure 8 shows an example of two correspondence point clouds with the corresponding dendrograms.

\subsection{Loop Closure: Local vs. Global Landmarks}

Loop closure, considered as one of the challenging issues in the robustness and scalability of SLAM
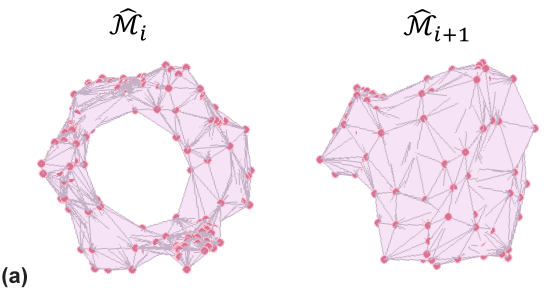

(b)

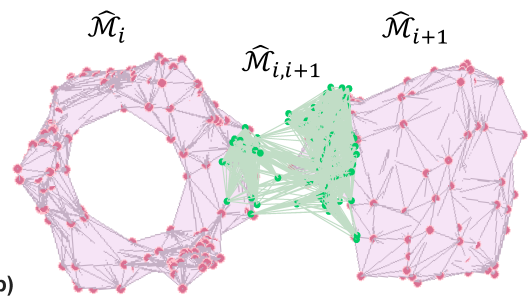

Figure 7: Map merging: (a) Two partial simplicial maps obtained from local point clouds $\hat{\mathcal{M}}_{i}$ and $\hat{\mathcal{M}}_{i+1}$, (b) the correspondence point cloud $\hat{\mathcal{M}}_{i, i+1}$ stitching them together for a global map.
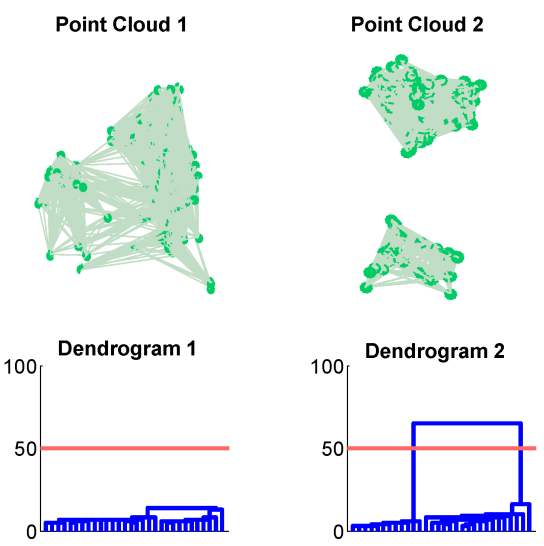

Figure 8: Single linkage clustering for two correspondence point clouds drawn from simulation results in Figure 10: (left) a one cluster point cloud versus (right) a two cluster point cloud.

techniques, refers to the data association task of deciding whether or not a local map being constructed is associated with a previously mapped regions $[79,80]$. This is usually transformed into asserting if a robot is revisiting a previously mapped area [81]. In cooperative SLAM, map intersection detection can be considered a loop closure problem where one agent is closing the loop built by another agent [82]. Here, however, loop closure can be viewed in terms of local mapping windows im- 

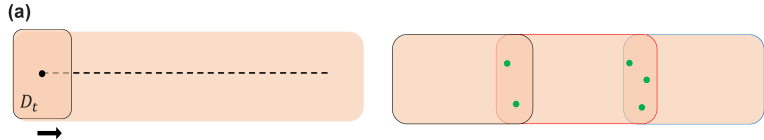

(b)

- Local landmarks Global landmark
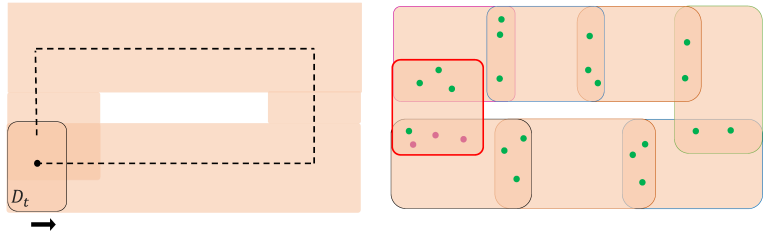

Figure 9: Two different scenarios where trajectories and coverage domains of the UAV are shown in the left, and mapping windows as well as landmarks are presented on the right side (local and global landmarks are plotted as green and pink dots, respectively): (a) a scenario with non-intersecting straight trajectory where local landmarks are sufficient for map-merging, (b) a scenario with loop closure where local landmarks are sufficient for merging temporally consecutive patches but global landmarks/features are required for loop closure detection.

posed by the leader, which can be transformed into a simpler problem of closing the loops in the trajectory of the leader (see Figure 9).

The methodology proposed here for joining local maps assumes spatially adjacency only between those maps that are temporally adjacent. This assumption is valid for scenarios where the L-UAV sweeps along non-intersecting trajectories. A direct generalization of this approach to cases with loop-closure is to make use of existing landmarks on the environment (e.g. using signals of opportunity [43]) or to augment the environment with permanently-static landmarks deployed over the entire domain. Detailed development of loop closure techniques for such scenarios is out of scope of this work, and requires further investigation. Figure 9 illustrates two different scenarios. Local landmarks are sufficient in the first scenario (case (a)). For the second scenario, global landmarks are required for stitching overlapping regions that do not intersect temporally but occur due to loop closure (case (b)).

\section{Experiments}

In this section, we present numerical as well as physical experimental results in order to demonstrate the validity of our dynamic GTP-Mapping approach. Videos of the simulations, physical experiment, and supplementary materials are avail- able in the project website ${ }^{1}$. Results demonstrating the local map construction for simpler scenarios can be found in [11].

\subsection{Numerical Experiments}

For our numerical simulation experiments, we have considered two-dimensional environments with basic geometric shapes for simplicity. However, the approach makes no use of these assumption and is directly applicable to more complex and unstructured environments. The software is composed of a swarm simulator and a topological mapping package. The swarm simulator (in $\mathrm{C}++$ ) reproduces the behavior of biobots by combining natural as well as controlled motion patterns. The topological mapping package (in $\mathrm{C}++$ and Matlab) performs metric estimation and topological recovery.

In Figure 6, we illustrated an example of numerical simulation results for construction of local GTP-Maps, and a variety of other examples can also be found in $[9,11]$. Here we present three different scenarios for global GTP-Map construction, for which the layouts of environments and the results are presented as shown in Figures 10 and 11. The objective of these simulations is to verify the correctness of the local as well as global GTPMapping procedures. In particular, Figure 10(eh) illustrates a scenario in which the map merging correctly stitches the local maps, even when the network constructed over the local domains is not connected.

In the swarm simulator, CRW parameters are set to be $C=1, l^{*}=3 m, v_{m}=1 \mathrm{~m} / \mathrm{s}$. All the ground agents are in CRW mode unless selected as landmarks. The change of direction is isotropic everywhere except at the boundaries of the coverage regions, where the agents change their direction towards the inside of the coverage domain of the LUAV. The L-UAV is considered to move along a straight line trajectory with a constant linear velocity of $v_{x}=0.05 \mathrm{~m} / \mathrm{s}$ in the $x$-direction. The coverage window of the aerial vehicle, for simplicity, is considered to be a rectangular arena with a length of $l=30 \mathrm{~m}$, and the same width as the environment. The dimensions of the environments are all set to be $100 \mathrm{~m} \times 300 \mathrm{~m}$. In our simulations, we assume an ideal communication channel among the

\footnotetext{
${ }^{1}$ https://research.ece.ncsu.edu/aros/project/ cyborg-insect-network/
} 
(a)

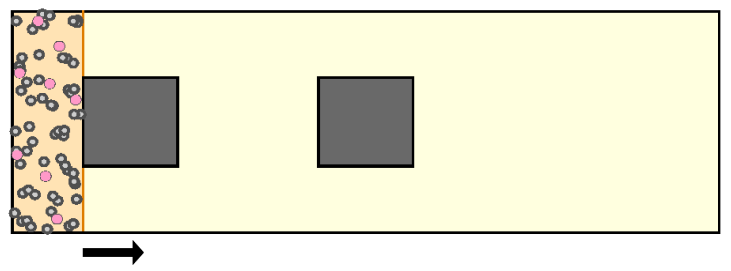

(b)

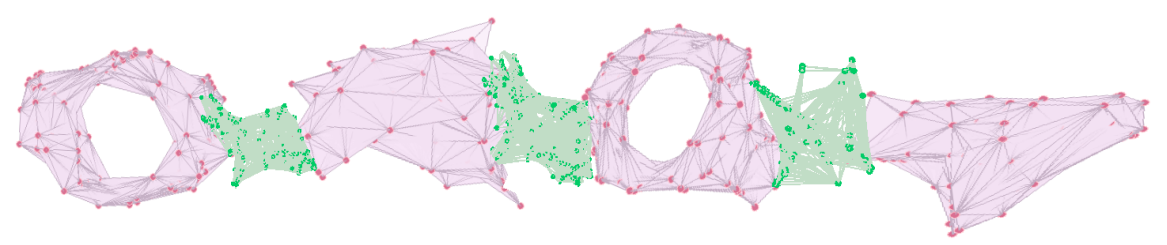

(c)
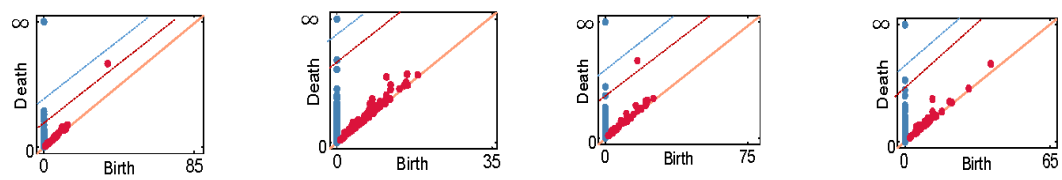

(d)

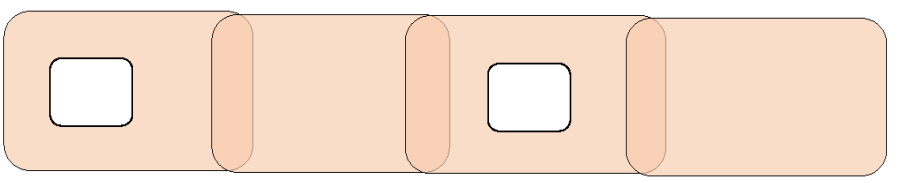

(e)

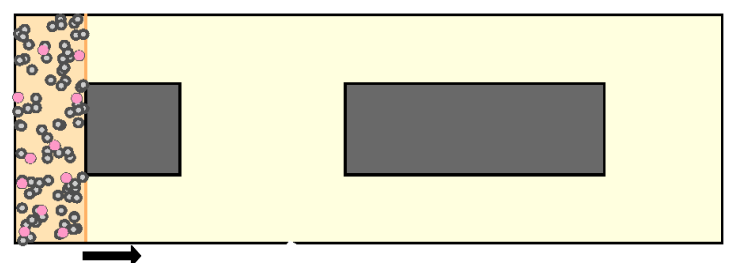

(f)

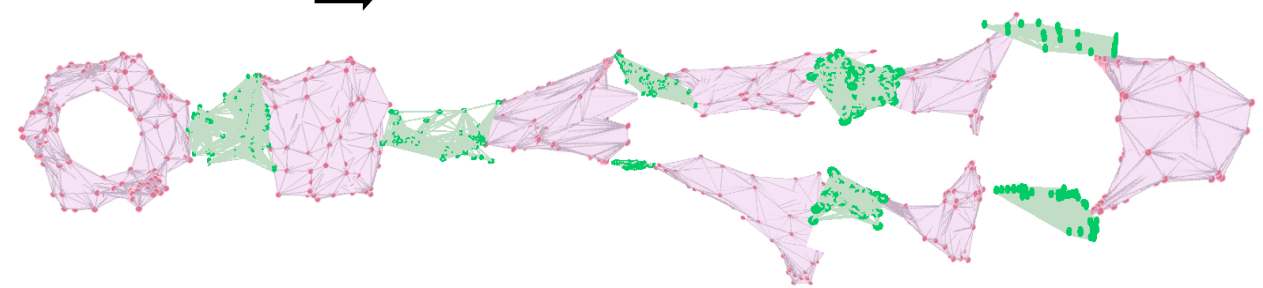

(g)
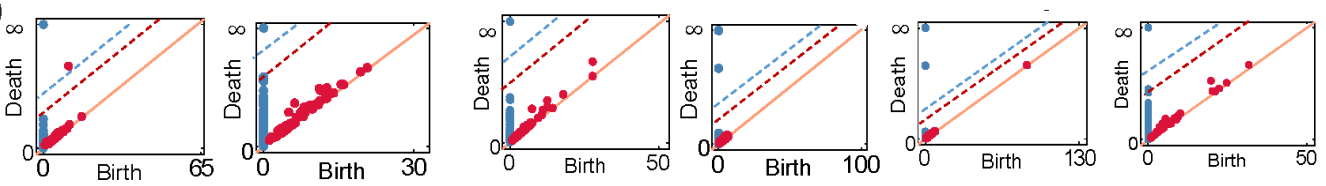

(h)

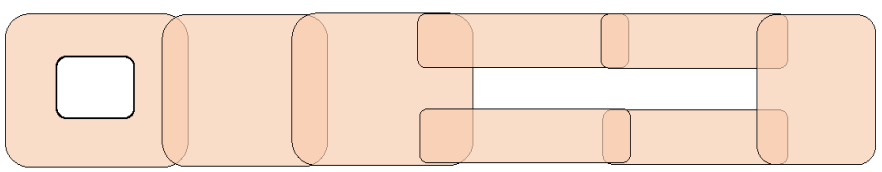

Figure 10: Simulation results for scenarios $1(\mathrm{a}-\mathrm{d})$ and $2(\mathrm{e}-\mathrm{h})$ : (a, e) The environments with initial configuration of agents for a sample run, (b, f) simplicial local maps (in pink) merged with correspondence complexes (green), (c, g) persistence diagrams with selected classification thresholds, and (d, h) synthetic global maps. 
(a)

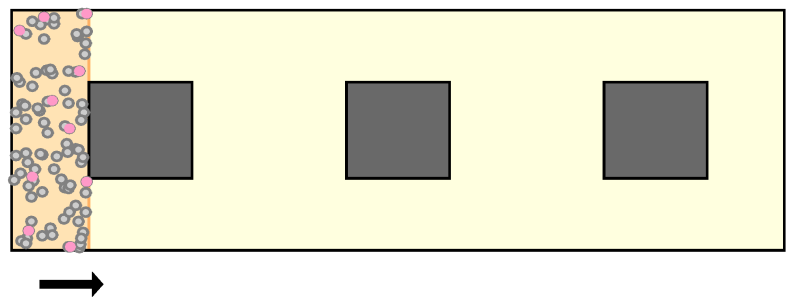

(b)

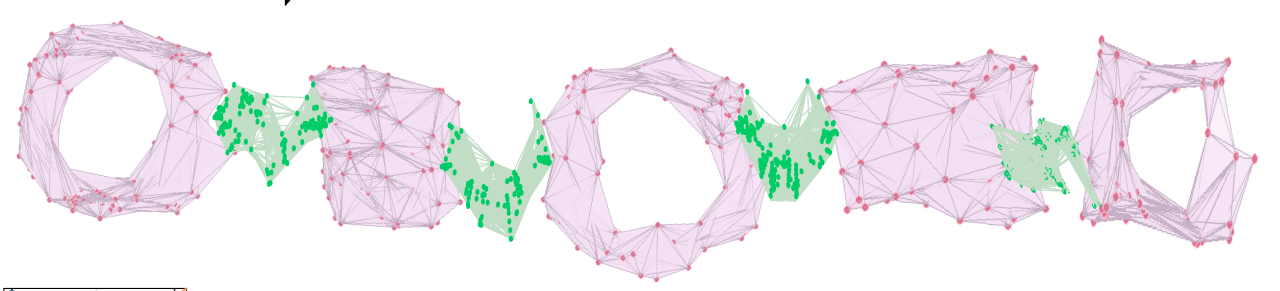

(c)
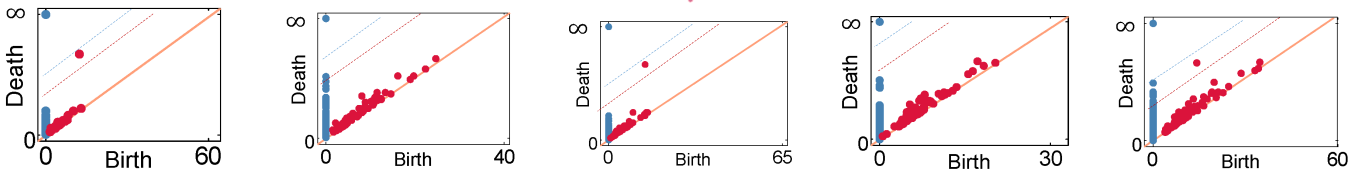

(d)

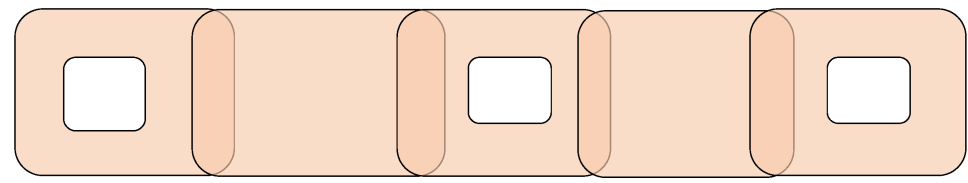

Figure 11: Simulation results for scenario 3: (a) The environment with initial configuration of agents for a sample run, (b) simplicial local maps (in pink) merged with correspondence complexes (green), (c) persistence diagrams with selected classification thresholds, and (d) a synthetic global map

agents as well as between the agents and the leader for simplicity. We will discuss the effect of communication constraints such as packet dropouts and delays on the system performance in section 11 .

The first row of each figure shows the physical environments together with the initial conditions of the agents (gray circles) at $t=0$ as well as the initial set of landmark nodes (shown as pink dots). For each scenario, a total number of 100 agents are considered in the network, among which $10 \%$ are selected as landmarks after some initial dispersion based on the maxmin landmark selection algorithm. The detection radius of each agent for encounter and boundary recognition is adopted to be $r_{d}=1 \mathrm{~m}$.

We divide the whole time window into 4,6 , and 5 overlapping windows for scenarios 1,2 , and 3 , respectively. Note that such selection has been made for sake of better visualization of the features, but in general we could select the same number of windows for all scenarios. Figures 10(b), 10(f), and 11(b) represent the local simplicial maps obtained for each scenario. Each of these maps consist of Vietoris-Rips complexes constructed on local estimated point clouds (shown in pink), which are in turn stitched together using the correspondence point clouds (shown in green) in order to obtain a global topological representations of each environment. The obstacles in the physical environments are represented as holes in these representations. To specify the number of clusters for each point cloud, a clustering threshold value of $\tau=50$ has been selected experimentally for all scenarios. In all cases, except the last three point clouds in scenario 2 , the correspondence point clouds are classified as one cluster. In scenario 2 , as a result of the large obstacle, local maps $\mathcal{M}_{4}$ and $\mathcal{M}_{5}$ and the merging point clouds are all divided into two clusters.

The persistence diagrams summarizing topological features of each local simplicial map are depicted in Figures 10(c), 10(g), and 11(c). On each persistence diagram, 0-dimensional and 1-dimensional topological features (connected components and 


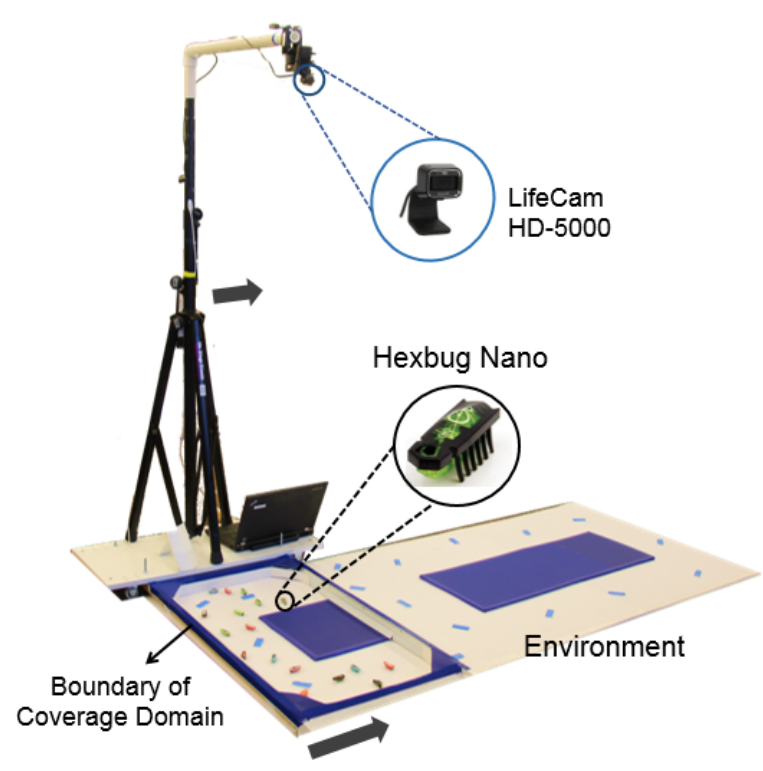

Figure 12: An overview of the physical setup for GTPmapping experiments with a swarm of Hexbug Nanos.

holes) are plotted as "blue" and "red" points, respectively, and the corresponding thresholds for the persistence classifiers are sketched as dashed lines. The values of thresholds are obtained using the classification method of section 8 as $\tau_{0}=25, \tau_{1}=30$. It can be observed that the point clouds representing the local maps capture the correct topological features of the local domains covered over each window. This can be verified by the topological features classified as robust ones in the corresponding persistence diagrams. Moreover, the estimated point clouds also contain some geometric information, which could be exploited to improve the mapping accuracy for approaches that make use of additional sensing and localization information. Finally, Figures 10(d), 10(h), and 11(d) show the global synthetic maps for each environment. They can be used as an approximate and for intuitive visualization of the space. Additionally, the topological information can be used as a constraint to ensure that a metric reconstruction properly captures connectivity in the space as in traditional SLAM approaches.

\subsection{Physical Experiments}

In this section, we present experimental results using a swarm of micro robotic creatures, called Hexbug Nanos [13], which emulate the natural random walk motion of biobots.

\subsubsection{System Setup}

An overview of the components of our experimental setup is illustrated in Figure 12.

Bio-Inspired agents. The Hexbug Nano (see Figure 12) uses the physics of vibration to propel forward and explore the environment. Powered by a tiny motor and 12 fixed, angular rubbery legs, they crawl forward and quickly navigate through complex mazes. When coming into contact with objects (walls, obstacles, or other Hexbugs) in their path, the agents switch directions towards a new path due to their persistent random behavior.

Environment. A physical environment to be mapped is constructed as shown in Figure 12, consisting of $2.29 \mathrm{~m} \times 1.01 \mathrm{~m}$ foam board, with two obstacles (one short and one long) similar to the environment used for our second numerical simulation scenario. The rectangular obstacles (in dark blue) are of dimensions $36 \mathrm{~cm} \times 36 \mathrm{~cm}$ and $96 \mathrm{~cm} \times 36 \mathrm{~cm}$ respectively, and are built from the same material. Since the motion mode of Hexbugs cannot be controlled into static mode, a number of 17 static landmarks are attached to the environment (small rectangular objects in blue in a size comparable to Hexbugs). The locations of landmarks are selected randomly in the free space of the environment.

Leader System. Since the Hexbug Nanos are not capable of communication nor motion control, we have emulated the invisible boundary for biobots by constructing a moving cart system in order to replicate the role of the aerial leader for herding the swarm. A local physical boundary of dimensions $54.61 \mathrm{~cm} \times 97.80 \mathrm{~cm}$ is attached to the moving cart, which plays the role of the local mapping region. The local frame is moving with the cart, with a speed of $0.2 \mathrm{~cm} / \mathrm{sec}$ in the direction shown in Figure 12. When the Hexbugs are deployed as a group in the local bounded arena, their stochastic movement emulates the random walk motion of the insects.

Visual Tracking System. Due to lack of communication capabilities among the Hexbugs, we employ a visual tracking system to identify and record the encounter information between the ground agents. A Microsoft LifeCam HD-5000 Webcam [83] (Figure 12 ) is mounted over a frame attached to the moving cart for this purpose. Visual tracking is carried out through the following stages: (1) background removal, (2) agent segmentation, (3) camera tracking, and (4) encounter tracking. Each video frame 

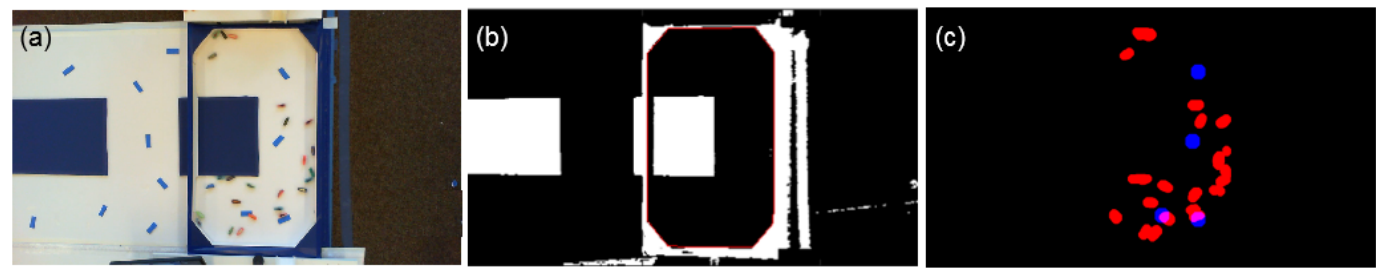

Figure 13: Visual tracking results for encounter detection shown for an example frame in the video: (a) An image capturing the local domain with Hexbugs moving stochastically therein and landmarks (shown in blue), (b) background image, and (c) regions corresponding to connected components (red for moving agents and blue for landmarks) identified via color segmentation.

is first converted into the Lab color space, and the background (including boundaries and obstacles) is extracted and removed from the frame via color segmentation. The connected components remaining after background removal (shown as red in Figure 13) correspond to the Hexbugs in the video. In order to track the location of the camera, we employ correspondence of the landmarks in the local frames with the world frame in order to compute the homography between these frames [84]. The encounter events are identified through a sequence of merges and splits of the connected components in the video. We record an encounter event whenever two connected components merge until they split, and measure the approximate elapsed time after the split till the next merge to construct the edge weights $w_{i j}$ in the encounter graph. Landmarks are detected as part of the color segmentation process, and the encounters with them are recorded and identified. Note that the tracking system is solely used for encounter identification, and no trajectory tracking nor coordinate information is recorded.

\subsubsection{Event Collection and Map Building}

Hexbugs start their motion from a random deployment inside the area located at one end of the environment as shown in Figure 12, and disperse through the arena for exploration. After an initial dispersion, the cart starts moving in the specified direction while the Hexbugs remain within the boundary of the moving region. At each time window, they efficiently explore the assigned sliding region. The encounter information is then exploited into our data analysis framework in order to estimate geometric point clouds and robustly find the topological structure of local areas.

\subsubsection{Experimental Results}

Figure 14 illustrates the results of our mapping experiment with the swarm of Hexbugs. Figure 14(a) shows the top view of the physical environment setup. For map construction, the whole time frame is divided into 4 overlapping windows for local mapping each with a duration of approximately 250 seconds. Figure 14(b) sketches a global simplicial map constructed from merging local subsampled point clouds obtained from coordinate free encounters using the proposed approach. The geometric point clouds for the local maps are very similar to the results of numerical simulation. The corresponding persistence diagrams and inferred topological features for each local window are plotted in Figure 14(c), with 0-dimensional and 1-dimensional features visualized as blue and orange dots, respectively. Persistence diagrams with classified features infer the existence of $1,2,1$, and 1 connected components for the local maps in Figure 14(b) from left to right, respectively. They further infer 0 persistence holes for the all point clouds except for the rightmost one, which matches the correct topological shape of the local domains. The inferred synthetic global map this experiment is plotted in Figure 14(d). It can be further verified from the plots that topological sketches inferred for each scenario are compatible with the structure of the physical environment.

\subsection{Comparison of Scales}

In order to illustrate how the results presented here scale to real world applications, we compare the geometric parameters used in our simulations and in the Hexbugs experiments, and how these parameters scale as a function of the characteristic velocities of the biobots. As discussed earlier, the bounds on metric uncertainty are dependent on the density of encounter samples in the environment, which in turn depend on the scale of the 
(a)

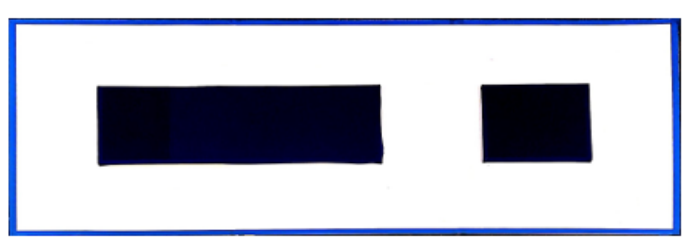

(b)

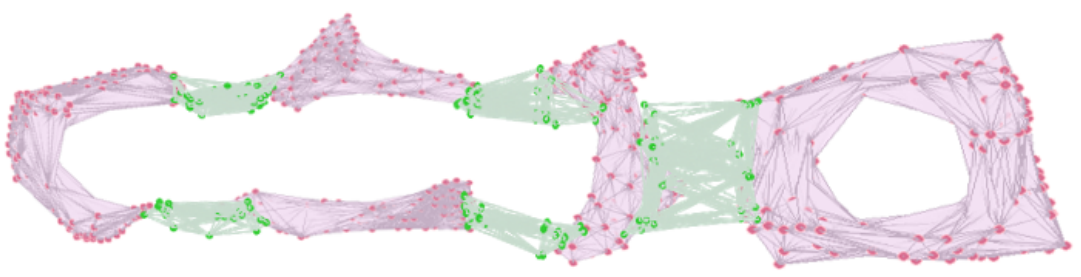

(c)
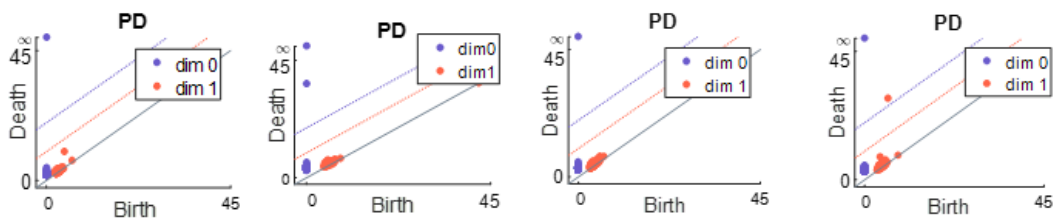

(d)

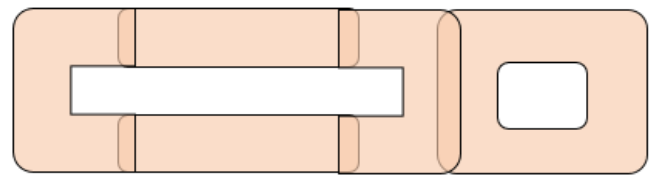

Figure 14: Experimental results for mapping with a swarm of Hexbugs (a) the top view of the physical environment, (b) geometric point clouds representing local maps (in pink) merged together to produce a global map (c) persistence diagrams representing the topological features of local maps, (d) an inferred synthetic map of the environment

Table 1: Comparison of scaling parameters used for the simulation with biobots (BioSim) and the experiments with Hexbugs (HB), and a scaled version of the experiments (Scaled).

\begin{tabular}{|l|c|c|c|c|c|}
\hline & Scale of $\mathcal{M}$ & $n_{m}$ & $v_{m}$ & $r_{d}$ & $v_{L}$ \\
\hline \hline BioSim & $100 \mathrm{~m}$ & 100 & $1 \mathrm{~m} / \mathrm{s}$ & $1 \mathrm{~m}$ & $5 \mathrm{~cm} / \mathrm{s}$ \\
\hline HB & $1 \mathrm{~m}$ & 20 & $0.25 \mathrm{~m} / \mathrm{s}$ & $0.05 \mathrm{~m}$ & $0.2 \mathrm{~cm} / \mathrm{s}$ \\
\hline Scaled & $100 \mathrm{~m}$ & 100 & $0.5 \mathrm{~m} / \mathrm{s}$ & $1 \mathrm{~m}$ & $2.5 \mathrm{~cm} / \mathrm{s}$ \\
\hline
\end{tabular}

environment $\mathcal{M}$, number of moving agents $\left(n_{m}\right)$, the velocity of agents $\left(v_{m}\right)$, and connectivity radius $\left(r_{d}\right)$. In [12] we present detailed experimental results for evaluation of metric estimation accuracy and how it varies with the number of agents. Table 1 provides a summary of the parameters used for the simulations (BioSim) and the experiments with the Hexbugs (HB). We note that the parameters for the simulations were selected to mirror a realistic deployment with the biobots. In particular, a number of 100 agents are considered to cover a region with a width of $100 \mathrm{~m}$. A velocity of $0.5 \mathrm{~m} / \mathrm{s}$ is considered for the biobots, which is under the maximum speeds of $5.4 \mathrm{~km} / \mathrm{h}$ reported for American Cockroaches [85]. A detection radius of $1 \mathrm{~m}$ is considered for robustly detecting neighboring biobots, and a velocity of $5 \mathrm{~cm} / \mathrm{s}$ is considered for the L-UAV. In order to scale these experiments to a scenario which mirrors better the physical constraints that biobots could experience in real deployments with obstacles (see Scaled row in Table 1), we have to choose a smaller maximum velocity of biobots due to the terrain constraints (e.g. $0.5 \mathrm{~m} / \mathrm{s}$ ). As a result, we would have chosen a slower leader velocity $(2.5 \mathrm{~cm} / \mathrm{s})$ accordingly. In this case the running time of the experiment needs to be increased by a factor of two as well in order to maintain the amount of total area coverage and the total density of the encounter samples gathered over time. One can alternatively choose a larger detection radius for the agents (e.g. $r_{d}=5 \mathrm{~m}$ ) in order to compensate for lower velocities. The speed 
Table 2: Average processing times for different stages of local mapping in scenario 1 of the numerical experiments for 20 independent runs (all values are measured in seconds). The values are reported per sliding windows of lengths 150 seconds in the experiments, for which the average total processing time for mapping is 33.18 seconds. This can be reduced considerably by means of parallelization and downsampling.

\begin{tabular}{|ccccc|ccc|}
\hline \multicolumn{4}{|c|}{ Metric Estimation } & \multicolumn{3}{c|}{ Top. Estimation } \\
\hline \hline EDP & EG & GSP & DR & Total & SS & PH & Total \\
\hline 1.97 & 0.59 & 19.76 & 5.30 & 27.62 & 3.71 & 1.85 & 5.56 \\
\hline
\end{tabular}

of the L-UAV, however, can be increased if we consider simple strategies such as first exploring for a specific time window, then switch to a pure herding behavior for another interval, and then switching to an exploration phase again.

\section{Discussion}

In this section we discuss issues related to computational complexity, developing a decentralized implementation, network constraints considerations, and integration with existing SLAM approaches.

\subsection{Computational Complexity}

Computational demand of mapping techniques is one of the determining factors to be considered for feasibility of real time implementations [86]. One of the drawbacks of the prior work [9] was its computational cost, which prevented scaling to large mapping scenarios. Dividing the area of interest into local regions to be mapped and then joining the maps reduces the computational run-time of the mapping procedure to a great extent, and is expected to yield roughly constant map updates per local map region. Looking carefully in the local mapping pipeline, the main computational blocks of our algorithm consists of: Event Detection and Processing (EDP), metric estimation (including Encounter Graph construction (EG), Graph Shortest Path (GSP) algorithm, Dimension Reduction (DR)), and topological estimation (including Subsampling (SS) and Persistence Homology $(\mathrm{PH})$ ). In order to evaluate computational cost for each block, we have measured elapsed time for each stage of map construction for 20 independent runs of the example scenario 1 in the numerical experiments. The processing times averaged over the four local windows and all independent runs are summarized in Table 2. We observed that the main bottlenecks in processing data were the large computation times for calculations of graph shortest path in metric estimation, dimension reduction, and persistent homology. Adding new events to the encounter graph increases its size by adding nodes and edges as well as the dimension of the corresponding weight matrix. This will make graph calculations very expensive and impractical with unbounded growth of the number of events and corresponding matrices over time. In particular, computational complexity of graph shortest path algorithm for directed graphs is $\mathcal{O}(V E)$, where $V$ and $E$ are the number of the vertices and the edges in the graph, respectively [87]. This will make metric estimation to be $\mathcal{O}\left(n^{3}\right)$ in the worst case with respect to the number of events $n$. One can benefit from parallel computing tools for graph algorithms in order to reduce computational cost of this stage. The computational complexity for persistent homology is reported to be $\mathcal{O}\left(m^{w}\right)$, in the worst case, where $w \approx 2.73$ and $m$ being the number of nodes in the point cloud [88]. On the other hand, with growing dimensions of the space, in order to preserve the sampling density conditions, dimension of the samples in the point cloud grow quadratically with the length scale, which will make persistence computations impractical for the entire point cloud. In order to deal with this issue, the subsampling strategy in 8.1 is acquired, and in our experiments the cardinality of each point cloud is set to a limit of $n_{S}$ (e.g. 500) data points. However, for larger environments, a small number of points will not be sufficient to recover topological and geometric features of the space accurately. Alternatively, we could use witness complexes instead of Rips complexes [9], which are less robust to outliers. These all make the original method of [9] unscalable and impractical for complex environments. Hence, the hierarchical approach presented here is a step towards a scalable mapping system, as it yields roughly constant updates per local map region. Note that map merging computations is dominated by graph shortest path calculations in the intersection windows, which is in the same order of computational complexity as the local window processing. With enough density of landmarks, one can select arbitrary small windows of local regions, and limit the number of subsampled events per window, in order to maintain accuracy of estimation while preventing large growth of computational complexity. 


\subsection{Decentralized Implementation}

Approaches for mapping unknown environments can be categorized as online or offline methods [80]. While most online solutions only use current observations for updating the map assuming temporal independence, offline methods often take all the past and present information into the account for building a map. It is noteworthy to mention that local mapping in our methodology is an offline approach over the local regions by its nature, as all the encounter information within a certain window are required to be processed to build the local metric. At a global level, on the other hand, only the last two consecutive windows are needed for map merging by processing their intersections.

However, a decentralized implementation of the local map along with parallel processing for graph algorithm computations can pave the way towards real time map construction [71]. On this note, one can realize that our proposed framework does not require connectivity of the communication network within the nodes in the entire network. In a basic setup, the biobots can create an ad-hoc network in which the agents are connected only when they are within a specific range. Each agent stores a list of its own encounter events locally and shares it with their neighbors on the go. Hence, information processing over the events for metric construction and persistent homology computation can be carried out either in a centralized or decentralized manner. In the centralized framework (implemented in this paper), all of the encounter information is sent to a centralized node (in this case the L-UAV) via a multi-hop connection. Then metric estimation and point cloud construction are processed in the central node over specific temporal windows, over which topological persistence is computed. In this scenario, the biobotic nodes act as passive agents, and do not require any on-board processing power. Temporal relationships required for metric estimation can be acquired from a time synchronization scheme appropriate for sparse ad-hoc networks [89]. Note that a global time synchronization is not required and only relative temporal information over local windows suffice. Alternatively, in the decentralized architecture, the ground agents can locally process the metric construction within a neighborhood in the ad-hoc network and track construction of simplices. Likewise, computation of homology groups and persistence can be carried out in a decentralized manner. Decentralized computation for homological features in networks is addressed in [90], and algorithms for distributed computation of persistent homology can be found in [91]. An advantage of the decentralized method is that the ground agents do not require to send information to the aerial access point as the data processing is carried out distributedly; hence, a one-way communication channel from the UAV to the ground agents (to create the invisible fence) would be sufficient.

\subsection{Network Constraints}

Due to constraints in communication channels, networked systems are prone to problems such as packet dropouts, time delays, quantization, and synchronization, which makes robust information exchange between nodes more difficult, and may degrade the overall system performance [92]. Time delays due to processing delays in the nodes, the network access delay, or transmission delays in the channel are stochastic in most of the networks. Generally, they can be categorized as constant versus time-varying, and deterministic versus stochastic, with delays smaller or larger than the sampling rate. Packet dropouts, which may occur due to long transmitter delays or failures and network congestion, can be modeled as stochastic processes as well [93]. Severe consecutive packet losses develop into the disconnection of a network. Although in the proposed framework we have omitted such communication constraints and have made an assumption of ideal channels, as discussed earlier, we do not require full connectivity of the network hence intermittent disconnection will not have a major impact on the performance. Furthermore, as map estimation is not dependent on individual events (rather on a set of encounters) nor do we require online data transmission for control of the agents, the nodes can wait until they receive the next packet as long as stochastic latencies or packet dropouts are not persistent nor spatially correlated to a specific location. However, if consecutive packet dropouts and disconnections occur over a spatially correlated region of the environment, metric reconstruction for that portion might be incorrect. Acquiring local storage for biobotic agents with decentralized implementations will be a solution in which the agents need to transmit data less often, which will be more robust to such disconnections.

\subsection{Integration with SLAM Approaches}

Our proposed approach can be applied to underground mapping scenarios in which biobotic insects are assigned to explore potential underground 
regions, where no localization is provided (refer to Figure 1). The underground agents can communicate with the UAV through the links established with the agents above the ground. Furthermore, one can reconsider the sensing and localization constraints for the leader and consider an aerial agent which can carry higher bandwidth and sensors for localization (e.g. a camera or GPS). In this case, the proposed approach can be extended to fuse traditional localization and mapping techniques above the ground (e.g. network SLAM [94] or visual SLAM [77]) with the GTP-Maps underground in order to construct a more accurate map. The topological information extracted also can be used as constraints on connectivity (i) to evaluate metric reconstruction, (ii) to be integrated with traditional topological maps by constructing connectivity graph maps [47, 48], and (iii) as constraints in graph optimization for SLAM techniques. These are only a few examples of how our mapping technique can be integrated with traditional SLAM approaches to construct hybrid maps. Our current investigation on integration of IMU sensors into biobotic backpacks will also pave the ground towards the integration of our approach with IMU based localization and mapping techniques.

\section{Conclusion and Future Work}

In this work, we presented our methodology for mapping using biobotic networks with weak localization information. We estimate local and global metrics and infer topological features of unknown environments based on only binary proximity information from agents who stochastically explore sub-domains of the environment. We exploited controlled motion of biobots to provide local landmark functionalities with a small portion of them acting in static mode, while following a leading agent by maintaining their connectivity for global exploration. We employed manifold learning techniques and topological persistence to build and classify local maps and proposed an approach to merge them into global maps. Although the obtained maps will not be as accurate as the ones constructed when higher bandwidth sensing (e.g. visual sensing) or localization information is available, they will provide a robust summary of the space and constraints within. Such maps can be used for identifying local and global homology, an approximate geometric representation and intuitive visualization of the space, or as a constraint to ensure that a metric reconstruction properly captures connectivity in the space. We are currently investigating motion and behavioral analysis of our biobotic platform in response to neurostimulation signals towards development of reliable testbeds. Future work includes conducting mapping experiments with life biobotic swarms once their control and prediction mechanism perform robustly in order to allow for the realization of such networks. Transition between different environments (e.g. underground and aboveground) will be an interesting problem to tackle, which will bring its own challenges into play, and can be investigated in future work.

\section{Acknowledgment}

The authors would like to thank the reviewers for their invaluable feedback, which greatly improved the paper. Additionally, they would like to thank Boxuan Zhong, Junyu Chen, Charles Hirsch, and Yang Yue for their help with conducting experiments.

\section{References}

[1] D. Haldane, K. Peterson, F. Garcia Bermudez, R. Fearing, Animal-inspired design and aerodynamic stabilization of a hexapedal millirobot, in: Robotics and Automation (ICRA), 2013 IEEE International Conference on, 2013, pp. 3279-3286. doi:10.1109/ICRA.2013.6631034.

[2] T. Latif, A. Bozkurt, Line following terrestrial insect biobots, in: Engineering in Medicine and Biology Society (EMBC), 2012 Annual International Conference of the IEEE, IEEE, 2012, pp. 972-975.

[3] T. Latif, E. Whitmire, T. Novak, A. Bozkurt, Towards fenceless boundaries for solar powered insect biobots, in: Engineering in Medicine and Biology Society (EMBC), 2014 36th Annual International Conference of the IEEE, IEEE, 2014, pp. 1670-1673.

[4] A. S. Huang, A. Bachrach, P. Henry, M. Krainin, D. Maturana, D. Fox, N. Roy, Visual odometry and mapping for autonomous flight using an rgb-d camera, in: International Symposium on Robotics Research (ISRR), 2011, pp. 1-16.

[5] P. Henry, M. Krainin, E. Herbst, X. Ren, D. Fox, Rgbd mapping: Using kinect-style depth cameras for dense $3 \mathrm{~d}$ modeling of indoor environments, The International Journal of Robotics Research 31 (5) (2012) 647-663.

[6] B. Ferris, D. Fox, N. Lawrence, WiFi-SLAM Using Gaussian Process Latent Variable Models., IJCAI (2007) 2480-2485.

[7] D. Kurth, G. Kantor, S. Singh, Experimental results in range-only localization with radio, in: Intelligent Robots and Systems, 2003.(IROS 2003). Proceedings. 2003 IEEE/RSJ International Conference on, Vol. 1, IEEE, 2003, pp. 974-979. 
[8] H. M. Choset, Principles of robot motion: theory, algorithms, and implementation, MIT press, 2005.

[9] A. Dirafzoon, E. Lobaton, Topological mapping of unknown environments using an unlocalized robotic swarm, in: Intelligent Robots and Systems (IROS), 2013 IEEE/RSJ International Conference on, 2013, pp. 5545-5551. doi:10.1109/IROS.2013.6697160.

[10] H. Edelsbrunner, J. Harer, Computational Topology: an Introduction, American Mathematical Society, 2010.

[11] A. Dirafzoon, J. Betthauser, J. S. D. Benavides, E. Lobaton, Mapping of Unknown Environments using Minimal Sensing from a Stochastic Swarm, in: Intelligent Robots and Systems (IROS), 2014 IEEE/RSJ International Conference on, 2014, pp. 3842-3849.

[12] A. Dirafzoon, A. Bozkurt, E. Lobaton, Geometric Learning and Topological Inference with Biobotic Networks, IEEE Transactions on Signal and Information Processing over Networks (To Appear).

[13] Hexbug micro robotic creatures, http://www.hexbug. $\mathrm{com} / \mathrm{nano} /$.

[14] H. Durrant-Whyte, T. Bailey, Simultaneous localization and mapping: part i, Robotics Automation Magazine, IEEE 13 (2) (2006) 99-110. doi:10.1109/MRA.2006.1638022.

[15] F. Endres, J. Hess, J. Sturm, D. Cremers, W. Burgard, 3-D mapping with an rgb-d camera, Robotics, IEEE Transactions on 30 (1) (2014) 177-187. doi:10.1109/TRO.2013.2279412.

[16] B. Kim, M. Kaess, L. Fletcher, J. Leonard, A. Bachrach, N. Roy, S. Teller, Multiple relative pose graphs for robust cooperative mapping, in: Robotics and Automation (ICRA), 2010 IEEE International Conference on, IEEE, 2010, pp. 3185-3192.

[17] R. Kurazume, S. Oshima, S. Nagakura, Y. Jeong, Y. Iwashita, Automatic large-scale three dimensional modeling using cooperative multiple robots, Computer Vision and Image Understanding.

[18] N. Waniek, J. Biedermann, J. Conradt, Cooperative slam on small mobile robots, in: 2015 IEEE International Conference on Robotics and Biomimetics (ROBIO), IEEE, 2015, pp. 1810-1815.

[19] K. Y. Leung, T. D. Barfoot, H. H. Liu, Decentralized cooperative slam for sparsely-communicating robot networks: A centralized-equivalent approach, Journal of Intelligent \& Robotic Systems 66 (3) (2012) 321-342.

[20] D. Benedettelli, A. Garulli, A. Giannitrapani, Cooperative slam using m-space representation of linear features, Robotics and Autonomous Systems 60 (10) (2012) 1267-1278.

[21] C. Nieto-Granda, J. G. Rogers, H. I. Christensen, Coordination strategies for multi-robot exploration and mapping, The International Journal of Robotics Research (2014) 0278364913515309.

[22] R. Aragues, J. Cortes, C. Sagues, Distributed consensus on robot networks for dynamically merging featurebased maps, Robotics, IEEE Transactions on 28 (4) (2012) 840-854.

[23] L. Paull, G. Huang, M. Seto, J. J. Leonard, Communication-constrained multi-auv cooperative slam, in: Robotics and Automation (ICRA), 2015 IEEE International Conference on, IEEE, 2015, pp. 509-516.

[24] R. Jeanson, C. Rivault, J.-L. Deneubourg, S. Blanco, R. Fournier, C. Jost, G. Theraulaz, Self-organized aggregation in cockroaches, Animal Behaviour 69 (1)
(2005) 169-180. doi:10.1016/j.anbehav.2004.02.009.

[25] S. Garnier, J. Gautrais, M. Asadpour, C. Jost, G. Theraulaz, Self-Organized Aggregation Triggers Collective Decision Making in a Group of Cockroach-Like Robots, Adaptive Behavior 17 (2) (2009) 109-133. doi:10.1177/1059712309103430.

[26] K. a. Daltorio, B. R. Tietz, J. a. Bender, V. a. Webster, N. S. Szczecinski, M. S. Branicky, R. E. Ritzmann, R. D. Quinn, A stochastic algorithm for explorative goal seeking extracted from cockroach walking data, 2012 IEEE International Conference on Robotics and Automation (2012) 2261-2268doi:10.1109/ICRA.2012.6225020.

[27] F. Cao, C. Zhang, H. Y. Choo, H. Sato, Insectcomputer hybrid legged robot with user-adjustable speed, step length and walking gait, Journal of The Royal Society Interface 13 (116) (2016) 20160060.

[28] F. Cao, C. Zhang, H. Y. Choo, H. Sato, Insect-machine hybrid robot: Insect walking control by sequential electrical stimulation of leg muscles, in: Robotics and $\mathrm{Au}-$ tomation (ICRA), 2015 IEEE International Conference on, IEEE, 2015, pp. 4576-4582.

[29] G. Li, D. Zhang, Brain-computer interface controlled cyborg: Establishing a functional information transfer pathway from human brain to cockroach brain, PloS one 11 (3) (2016) e0150667.

[30] C. J. Sanchez, C.-W. Chiu, Y. Zhou, J. M. González, S. B. Vinson, H. Liang, Locomotion control of hybrid cockroach robots, Journal of The Royal Society Interface 12 (105) (2015) 20141363.

[31] J. Schwefel, R. E. Ritzmann, I. N. Lee, A. Pollack, W. Weeman, S. Garverick, M. Willis, M. Rasmussen, D. Scherson, Wireless communication by an autonomous self-powered cyborg insect, Journal of The Electrochemical Society 161 (13) (2015) H3113-H3116.

[32] H. Edelsbrunner, J. Harer, Persistent homology - a survey, Contemporary mathematics (2008) 1-26.

[33] P. Skraba, M. Ovsjanikov, F. Chazal, L. Guibas, Persistence-based segmentation of deformable shapes, in: Computer Vision and Pattern Recognition Workshops (CVPRW), 2010 IEEE Computer Society Conference on, IEEE, 2010, pp. 45-52.

[34] J. A. Perea, A. Deckard, S. B. Haase, J. Harer, SW1pers: Sliding windows and 1-persistence scoring; discovering periodicity in gene expression time series data, BMC bioinformatics 16 (1) (2015) 257.

[35] F. A. Khasawneh, E. Munch, Chatter detection in turning using persistent homology, Mechanical Systems and Signal Processing.

[36] P. Bendich, S. Chin, J. Clarke, J. deSena, J. Harer, E. Munch, A. Newman, D. Porter, D. Rouse, N. Strawn, et al., Topological and statistical behavior classifiers for tracking applications, arXiv preprint arXiv:1406.0214.

[37] J. A. Perea, J. Harer, Sliding windows and persistence: An application of topological methods to signal analysis, Foundations of Computational Mathematics 15 (3) (2013) 799-838.

[38] A. Dirafzoon, N. Lokare, E. Lobaton, Action Classification From Motion Capture Data Using Topological Data Analysis, in: 2016 IEEE Global Conference on Signal and Information Processing (GlobalSIP), IEEE, 2016.

[39] A. Tahbaz-Salehi, A. Jadbabaie, Distributed coverage verification in sensor networks without location information, Automatic Control, IEEE Transactions on 55 (8) (2010) 1837-1849. 
doi:10.1109/TAC.2010.2047541.

[40] V. de Silva, R. Ghrist, Coordinate-free Coverage in Sensor Networks with Controlled Boundaries via Homology, The International Journal of Robotics Research 25 (12) (2006) 1205-1222. doi: $10.1177 / 0278364906072252$.

[41] S. Kim, K. Sreenath, S. Bhattacharya, V. Kumar, Optimal trajectory generation under homology class constraints, in: Decision and Control (CDC), 2012 IEEE $51^{\text {st }}$ Annual Conference on, 2012, pp. 3157-3164.

[42] J. Derenick, A. Speranzon, R. Ghrist, Homological sensing for mobile robot localization, in: Robotics and Automation (ICRA), 2013 IEEE International Conference on, 2013, pp. 572-579. doi:10.1109/ICRA.2013.6630631.

[43] M. Robinson, R. Ghrist, Topological localization via signals of opportunity, Signal Processing, IEEE Transactions on 60 (5) (2012) 2362-2373.

[44] R. Ramaithitima, M. Whitzer, S. Bhattacharya, V. Kumar, Sensor coverage robot swarms using local sensing without metric information, in: Robotics and Automation (ICRA), 2015 IEEE International Conference on, IEEE, 2015, pp. 3408-3415.

[45] B. Walker, C. Park, Using persistent homology to recover spatial information from encounter traces, in: Proceedings of the $9^{\text {th }} \mathrm{ACM}$ international symposium on Mobile ad hoc networking and computing - MobiHoc '08, ACM Press, 2008, p. 371.

[46] R. Ghrist, D. Lipsky, J. Derenick, A. Speranzon, Topological landmark-based navigation and mapping, Tech. rep., University of Pennsylvania, Department of Mathematics (Aug. 2012).

[47] H. Choset, K. Nagatani, Topological simultaneous localization and mapping (SLAM): toward exact localization without explicit localization, Robotics and Automation, IEEE Transactions on 17 (2) (2001) 125-137. doi: $10.1109 / 70.928558$

[48] S. Tully, G. A. Kantor, H. Choset, A unified Bayesian framework for global localization and SLAM in hybrid metric/topological maps, International Journal of Robotics Research (IJRR) 31 (3) (2012) 271-288.

[49] H. Sato, M. M. Maharbiz, Recent developments in the remote radio control of insect flight, Frontiers in neuroscience 4 .

[50] D. Mellinger, V. Kumar, Minimum snap trajectory generation and control for quadrotors, in: Robotics and Automation (ICRA), 2011 IEEE International Conference on, IEEE, 2011, pp. 2520-2525.

[51] H. Chao, Y. Cao, Y. Chen, Autopilots for small unmanned aerial vehicles: a survey, International Journal of Control, Automation and Systems 8 (1) (2010) 3644.

[52] K. Anusuya, S. Bharadhwaj, S. S. Rani, Wireless channel models for indoor environments, Defence Science Journal 58 (6) (2008) 771-777.

[53] W. Cheng, M. Li, K. Liu, Y. Liu, X. Li, X. Liao, Sweep coverage with mobile sensors, in: Parallel and Distributed Processing, 2008. IPDPS 2008. IEEE International Symposium on, 2008, pp. 1-9. doi:10.1109/IPDPS.2008.4536245.

[54] R. Jeanson, S. Blanco, R. Fournier, J.-L. Deneubourg, V. Fourcassié, G. Theraulaz, A model of animal movements in a bounded space, Journal of Theoretical Biology 225 (4) (2003) 443-451. doi:10.1016/S00225193(03)00277-7.
[55] M. J. Plank, M. Auger-Méthé, E. A. Codling, Lévy or not? analysing positional data from animal movement paths, in: Dispersal, individual movement and spatial ecology, Springer, 2013, pp. 33-52.

[56] J. N. Twigg, J. R. Fink, P. L. Yu, B. M. Sadler, Rss gradient-assisted frontier exploration and radio source localization, in: Robotics and Automation (ICRA), 2012 IEEE International Conference on, IEEE, 2012, pp. 889-895.

[57] D. Kim, A. Liu, E. Diller, M. Sitti, Chemotactic steering of bacteria propelled microbeads, Biomedical microdevices 14 (6) (2012) 1009-1017.

[58] E. Chambers, V. Silva, J. Erickson, R. Ghrist, Vietoris-rips complexes of planar point sets, Discrete and Computational Geometry 44 (1) (2010) 75-90. doi:10.1007/s00454-009-9209-8.

[59] H. Edelsbrunner, D. Letscher, A. Zomorodian, Topological persistence and simplification, Discrete and Computational Geometry 28 (4) (2002) 511-533.

[60] A. Zomorodian, G. Carlsson, Computing persistent homology, Proceedings of the twentieth annual symposium on Computational geometry - SoCG '04 (2004) 347doi:10.1145/997817.997870.

[61] D. Horak, S. Maletić, M. Rajković, Persistent homology of complex networks, Journal of Statistical Mechanics: Theory and Experiment 2009 (03) (2009) P03034. doi:10.1088/1742-5468/2009/03/P03034.

[62] J. Beal, Superdiffusive dispersion and mixing of swarms, ACM Transactions on Autonomous and Adaptive Systems (TAAS) 10 (2) (2015) 10.

[63] V. De Silva, G. Carlsson, Topological estimation using witness complexes, Proc. Sympos. Point-Based Graphics (2004) 157-166.

[64] T. H. Cormen, C. Stein, R. L. Rivest, C. E. Leiserson, Introduction to Algorithms, 2nd Edition, McGraw-Hill Higher Education, 2001

[65] T. F. Cox, M. A. A. Cox, Multidimensional Scaling, 2nd Edition, Chapman and Hall / CRC, 2000.

[66] J. B. Tenenbaum, V. de Silva, J. C. Langford, A global geometric framework for nonlinear dimensionality reduction., Science (New York, N.Y.) 290 (5500) (2000) 2319-23. doi:10.1126/science.290.5500.2319.

[67] J. Zhang, H. Huang, J. Wang, Manifold learning for visualizing and analyzing high-dimensional data, Intelligent Systems, IEEE 25 (4) (2010) 54-61. doi:10.1109/MIS.2010.8

[68] D. Cohen-Steiner, H. Edelsbrunner, J. Harer, Stability of persistence diagrams, Discrete \& Computational Geometry 37 (1) (2007) 103-120.

[69] A. Dirafzoon, A. Bozkurt, E. Lobaton, Geometric Learning and Topological Inference with Biobotic Networks: Convergence Analysis, arXiv preprint arXiv:1607.00051.

[70] J. Guivant, E. Nebot, J. Nieto, F. Masson, Navigation and mapping in large unstructured environments, The International Journal of Robotics Research 23 (4-5) (2004) 449-472.

[71] J. E. Guivant, E. M. Nebot, Optimization of the simultaneous localization and map-building algorithm for real-time implementation, IEEE transactions on Robotics and Automation 17 (3) (2001) 242-257.

[72] J. Nieto, J. Guivant, E. Nebot, A novel hybrid map representation for denseslam in unstructured large environments, Proc. IEEE International confrence on Robotics and Automation ICRA 1 (2004) 391-396. 
[73] B. T. Fasy, F. Lecci, A. Rinaldo, L. Wasserman, S. Balakrishnan, A. Singh, et al., Confidence sets for persistence diagrams, The Annals of Statistics 42 (6) (2014) 2301-2339.

[74] G. Carlsson, T. Ishkhanov, V. Silva, A. Zomorodian, On the Local Behavior of Spaces of Natural Images, International Journal of Computer Vision 76 (1) (2007) 1-12. doi:10.1007/s11263-007-0056-x.

[75] D. Morozov, Dionysus library for persistent homology, Available at http://mrzv.org/software/dionysus.

[76] S. Carpin, A. Birk, V. Jucikas, On map merging, Robotics and autonomous systems 53 (1) (2005) 1-14.

[77] P. Henry, M. Krainin, E. Herbst, X. Ren, D. Fox, Rgb-d mapping: Using depth cameras for dense 3d modeling of indoor environments, in: In the 12th International Symposium on Experimental Robotics (ISER, Citeseer, 2010.

[78] S. Theodoridis, K. Koutroumbas, Pattern Recognition, 4th Edition, Academic Press, 2008.

[79] P. Newman, K. Ho, Slam-loop closing with visually salient features, in: Robotics and Automation, 2005. ICRA 2005. Proceedings of the 2005 IEEE International Conference on, IEEE, 2005, pp. 635-642.

[80] F. Mutz, L. P. Veronese, T. Oliveira-Santos, E. de Aguiar, F. A. A. Cheein, A. F. De Souza, Largescale mapping in complex field scenarios using an autonomous car, Expert Systems with Applications 46 (2016) 439-462.

[81] K. L. Ho, P. Newman, Loop closure detection in slam by combining visual and spatial appearance, Robotics and Autonomous Systems 54 (9) (2006) 740-749.

[82] K. L. Ho, P. Newman, Detecting loop closure with scene sequences, International Journal of Computer Vision 74 (3) (2007) 261-286.

[83] Microsoft lifecam hd-3000, http://www.microsoft. com/hardware/en-us/p/lifecam-hd-3000/T3H-00011.

[84] Y. Ma, S. Soatto, J. Kosecka, S. S. Sastry, An invitation to 3-d vision: from images to geometric models, Vol. 26, Springer Science \& Business Media, 2012.

[85] https://en.wikipedia.org/wiki/American_ cockroach.

[86] J. Nieto, J. Guivant, E. Nebot, S. Thrun, Real time data association for fastslam, in: Robotics and Automation, 2003. Proceedings. ICRA'03. IEEE International Conference on, Vol. 1, IEEE, 2003, pp. 412-418.

[87] A. Schrijver, Combinatorial optimization: polyhedra and efficiency, Vol. 24, Springer Science \& Business Media, 2003.

[88] H. Edelsbrunner, D. Morozov, Persistent homology: theory and practice, in: Proceedings of the European Congress of Mathematics, 2012, pp. 31-50.

[89] K. Römer, Time synchronization in ad hoc networks, in: Proceedings of the 2nd ACM international symposium on Mobile ad hoc networking \& computing, ACM, 2001, pp. $173-182$.

[90] A. Muhammad, A. Jadbabaie, Decentralized Computation of Homology Groups in Networks by Gossip, 2007 American Control Conference (2007) 34383443doi:10.1109/ACC.2007.4283133.

[91] U. Bauer, M. Kerber, J. Reininghaus, Distributed computation of persistent homology., in: ALENEX, SIAM, 2014, pp. 31-38.

[92] L. Zhang, H. Gao, O. Kaynak, Network-induced constraints in networked control systemsa survey, Industrial Informatics, IEEE Transactions on 9 (1) (2013)
403-416.

[93] M. Sahebsara, T. Chen, S. L. Shah, Optimal filtering with random sensor delay, multiple packet dropout and uncertain observations, International journal of control 80 (2) (2007) 292-301.

[94] J. Djugash, S. Singh, Motion-aided network SLAM with range, Int. J. Rob. Res. 31 (5) (2012) 604-625. doi: $10.1177 / 0278364912441039$. 